\title{
NOEC and LOEC as merely concessive expedients: two unambiguous alternatives and some criteria to maximize the efficiency of dose-response experimental designs
}

\author{
M. A. Murado and M. A. Prieto* \\ Instituto de Investigacións Mariñas (CSIC) \\ r/ Eduardo Cabello, 6. 36208-Vigo. Galicia (Spain) \\ *Corresponding author: michaelumangelum@gmail.com
}

\begin{abstract}
NOEC and LOEC (no and lowest observed effect concentration, respectively) are toxicological concepts derived from analysis of variance (ANOVA), a not very sensitive method that produces ambiguous results and does not provide confidence intervals (CI) of its estimates. For a long time, despite the abundant criticism that such concepts have raised, the field of the ecotoxicology is reticent to abandon them (two possible reasons will be discussed), adducing the difficulty of clear alternatives. However, this work proves that a debugged dose-response (DR) modeling, through explicit algebraic equations, enables two simple options to accurately calculate the CI of substantially lower doses than NOEC. Both ANOVA and DR analysis are affected by the experimental error, response profile, number of observations and experimental design. The study of these effects -analytically complex and experimentally unfeasible- was carried out using systematic simulations with realistic data, including different error levels. Results revealed the weakness of NOEC and LOEC notions, confirmed the feasibility of the proposed alternatives and allowed to discuss the -often violated-conditions that minimize the CI of the parametric estimates from DR assays. In addition, a table was developed providing the experimental design that minimizes the parametric $\mathrm{CI}$ for a given set of working conditions. This makes possible to reduce the experimental effort and to avoid the inconclusive results that are frequently obtained from intuitive experimental plans.
\end{abstract}

Key words: NOEC and LOEC; ANOVA; dose-response models; modeling; confidence intervals; parametric estimations.

\section{INTRODUCTION}

The essential parameter for characterizing a toxic agent $\left(\mathrm{ED}_{50}\right.$ or $\mathrm{EC}_{50}$, dose or concentration producing the half-maximum response) is not very suitable for the ecotoxicological context, since it represents an excessive effect. Thus, NOEC (sometimes called NOAEL: non-observable adverse effect level) and LOEC seem to provide useful complementary concepts, reporting the detection limits of the applied method. These limits are routinely estimated through ANOVA, by comparing the responses of a control with those obtained for increasing concentrations of an agent in the threshold region, and the result is usually added as a key information to the $\mathrm{EC}_{50}$ value derived from some formal type of dose-response (DR) model. Despite numerous objections (Chapman et al. 1996; Fox et al. 2012; Kooijman. 1981;1996; Murrell et al. 1998; Pires et al. 2002; Warne M and Van Dam R. 2008), it is often said that these concepts are preserved due to the lack of alternatives or because the possible alternatives are complex, forcing researchers to abandon the standard DR approach through log-logistic or log-probit models (Kooijman and Bedaux. 1996). However, the practical application of such concepts is to replace the toxicological judgment by the idea of what is visibly detectable. At the same time, their use involve to renounce the results obtained from DR modeling and to favor a tool that, given the 
usual error of the assays in this field, is scarcely sensitive, extremely dependent on the experimental procedures and lacks of a criteria about its real accuracy.

By way of analogy, let us suppose that we are located on an asteroid, and we decide to estimate the gravity through the Galileo's experiment, by rolling balls along an inclined plane and measuring the time for covering increasing spaces. Once we have established from the model $s=g t^{2} / 2$ that the value of $g$ is, for example, $0.8 \mathrm{~m} . \mathrm{s}^{-2}$, we would not doubt -especially if some device of our return spaceship depends on it- that after $0.1 \mathrm{~s}$ from the starting time, all weight in free fall will have covered $4 \mathrm{~mm}$, even if our instruments do not allow us to verify it with an acceptable accuracy. As a result, a notion as «time corresponding to a non-observable space» would not be useful and probably most of us would run off from such a concept. Thus, the prominence of the concepts connected with the detection limits in ecotoxicology does not seem to translate a special attention to the precision, but rather to reveal distrust towards the modeling, or even towards the regularities of nature.

A tolerance limit should be established as a function of the toxic effect, and it is not unlikely to suppose that, in many cases, an effect that cannot be detected by ANOVA should be considered intolerable. An acute effect of $1 \%$ does not seem an exaggeration, but as it will be seen, it cannot be detected by ANOVA, even under conditions of low error and rigorous execution. Moreover, if the causal agent is metabolically recalcitrant, bioaccumulative, carcinogenic, able to interact with hormones or to link covalently to DNA, the $1 \%$ limit would seem unacceptably high.

Theoretically, any explicit algebraic DR model can predict the response to any dose, and it can be reparametrized in such a way that the dose corresponding to any specified response $(p)$ is an explicit parameter $\left(m_{\mathrm{p}}\right)$ of the expression, enabling the direct calculation of its confidence interval (CI). Although it represents an important advantage, the intrinsic variability of the response, the experimental error and sometimes inappropriate experimental designs can contribute to produce final results that are not much better than those derived from ANOVA.

However, we believe that the DR assays can be freed from some usual bugs and applied with a substantial control of the effects caused by the experimental error. When a suitable explicit model (with statistically significant parametric estimates and unbiased residuals) is available, at least two criteria can be defined to predict the response and quantify its variability in regions that, although appreciably lower than the NOEC value detected by ANOVA, are far from the toxicological irrelevance. This is a problem whose analytical focus overcomes us and whose experimental treatment would be unfeasibly laborious. Therefore, it will be examined here through "simulation experiments" under realistic conditions.

\section{THEORETICAL BACKGROUND AND METHODS}

\subsection{The dose-response model}

A DR model translates the distribution of the sensitivity to a toxic agent in a population and, therefore, a probability mass function represents the natural option among the equations able to describe the sigmoidal characteristic profile of this phenomenon. Although normal and lognormal distributions have been the basis for the traditional tools for DR analysis, they have the disadvantage of lacking in explicit forms in their mass functions. Logistic type equations are more useful and they can be written in DR-suitable forms (De Lean et al. 1978), but their derivatives (density functions) have only right bias. Another option is the Gompertz equation (Gompertz. 1825), but its reparametrized form when adapted to DR context becomes complex and difficult to manage. 
The Weibull distribution (Weibull. 1951), which is increasingly applied in DR analysis, is the common model for the failure of complex artifacts (to some extent a toxicity-isomorphic phenomenon), and it produces a varied family of curves that can describe satisfactorily diverse type of toxic effects (Murado et al. 2002; Murado and Vázquez. 2007; Murado and Vázquez. 2010; Prieto et al. 2012; Prieto et al. 2013; Riobó et al. 2008a; Riobó et al. 2008b; Vázquez et al. 2005). The Weibull mass function will be used multiplied by an asymptotic maximum $(K \leq 1)$, and reparametrized to make explicit the dose $\left(m_{\mathrm{p}}\right)$ that corresponds to any proportion $(p \leq 1)$ of the maximum response:

$$
R=K\left\{1-\exp \left[\ln q\left(D / m_{p}\right)^{a}\right]\right\} ; \text { being: } q=1-p
$$

or in its reciprocal form, which express the dose $(D)$ corresponding to a given response $(R)$ :

$$
D=m_{p}\{\ln [1-(R / K)] / \ln q\}^{1 / a}
$$

When $p=0.5$, if $K=1, m_{\mathrm{p}}=m_{0.5}=\mathrm{EC}_{50}$, and we will write simply $m$. It can be added that the shape parameter $(a)$ is related to the slope of the curve at the inflection point $\left(r_{\mathrm{m}}\right.$, at abscissa $\left.m\right)$, and to the maximum slope $\left(r_{\max }\right)$, through:

$$
\begin{aligned}
& r_{\mathrm{m}}=\frac{K a \ln 2}{2 m} \\
& r_{\max }=\frac{K a}{m}(\ln 2)^{1 / a} G^{G} \exp (-G) ; \text { siendo } G=\frac{a-1}{a}
\end{aligned}
$$

When $a=3.26\left(=a_{\mathrm{s}}\right)$ the function is symmetrical and $r_{\mathrm{m}}=r_{\max }$. If $a>a_{\mathrm{s}}$, the profile of the equation (1) approximates progressively to a step function in $m$. If $a<a_{\mathrm{s}}$, the abscissa of $r_{\max }$ is lower than $m$, and if $a=1$, equation (1) becomes the Bertalanffy equation, with the maximum slope at the origin. The $r_{\mathrm{m}}$ slope exists for any $a>0$, but $r_{\max }$ only for $a>1$ (notice the structure of the term $G$ ).

To facilitate comparisons, in all the cases we have considered that the response (and, unless otherwise stated, the concentration or dose as well) varies within the range $[0,1]$.

\subsection{Data generation without and with error}

In a previous work (Murado et al. 2002), DR relationships were simulated with an algorithm quantifying the response (death/survival) of a virtual cell population in the presence of increasing doses of a toxic agent. Each cell was described with two random numbers (any unimodal distribution with $[0, \infty)$ domain led to similar final consequences), which represented the number of receptors of the toxic per cell, and the threshold of the response or the number of receptors that should be occupied by one toxic unit for a lethal response. The results of this "constructivist" method were accurately described, under different hypotheses, by the Weibull equation. Therefore, the model (1) was directly applied to generate data series without error, using parametric values that were specified for each case.

The error in the dependent variable measurement can differ appreciably in different systems. If the response involves enzymatic assays or microbial cultures (especially bacteria and yeasts; fungi and algae can be more problematic), the accuracy is relatively high, but it decreases with the complexity of the biological entity, due to its own complexity and the less numerous target 
populations used. Even in larval growth inhibition and sperm bioassays with sea urchin (Lera et al. 2006; Novelli et al. 2003), coral or polychaetes (Negri and Heyward. 2001; Xie et al. 2005), the values of the standard deviation (usually considered homoscedastic) are around $10 \%$ of the half-maximum response.

Thus, in the simulation experiments that we will discuss next, the values resulting from equation (1) were modified by adding a normal homoscedastic error defined as (Box and Muller. 1958):

$$
N(0 ; \sigma)=\sigma\left[\left(-2 \ln u_{1}\right)^{1 / 2}\right] \sin \left(2 \pi u_{2}\right)
$$

where $u_{1}$ and $u_{2}$ are two uniform random numbers and $\sigma$ is the standard deviation of the normal error distribution. The values of $\sigma$ were in the range $[0.025,0.200]$, that is, $2.5-20 \%$ of the maximum response.

\subsection{Data analysis}

We define a virtual assay as the result from a simulation that, according to a macro written $a d$ hoc in a Microsoft Excel spreadsheet: 1) adds 2,000 times a random error to the responses produced by the model (1) with a given set of doses and parametric values; 2) performs at each time an ANOVA or a DR analysis as it is described next.

\subsubsection{ANOVA and NOEC-LOEC estimates}

By assigning values to the function (1) and using (1B) to calculate the dose corresponding to $1 \%$ of the maximum response, the resulting value $\left(D_{0.01}\right)$ was used as the unit of increasing series of doses defined as $D_{\mathrm{i}}=D_{(\mathrm{i}-1)}+n \times D_{0.01}$, where $n$ is a factor selected to obtain appropriate sets of 10 values (treatments). Next, the routine of ANOVA was achieved 2,000 times combining different values of $\sigma$ with the use of 3,6 and 10 replicates. Three criteria were applied in each run to decide the statistical significance of the mean differences: the absence of overlapping between CI derived from Student's $t$ distribution, and the Scheffé and Dunnett tests. Since the statistical significance was assessed with $\alpha=0.05$ in all cases, the first dose producing a significantly different response with regard to the control in $100 \%$ of the 2,000 repetitions was considered as the LOEC value, and NOEC the immediately precedent dose.

\subsubsection{DR analysis}

The responses produced by the function (1) with different parameters, dose series and $\sigma$ values were used to recalculate the starting expression. In this case the macro carried out: 1) the parameter estimation by non-linear least squares (quasi-Newton method), using the Solver complement included in the Microsoft Excel spreadsheet; 2) the determination of the parametric CI and the model consistence (Student and Fisher tests, respectively, with $\alpha=0.05$ in both cases), using Solver Aid (Prikler. 2009).

Since the parametric CI were calculated with $\alpha=0.05$ in the Student test, a virtual assay can be considered statistically significant (95\%) when $100 \%$ of the 2,000 repetitions are significant. Now then, the parametric CI can be obtained by two procedures: 1) by averaging directly the resulting $\mathrm{CI}$ from the 2,000 repetitions; 2) by calculating them (with $\alpha=0.05$ ) from the 2,000 parametric estimates. Although both criteria differ slightly, the second one is more concessive, and therefore the first one was chosen. Skewness and kurtosis coefficients of the parametric distributions were calculated using all the estimates, significant or not. Although they are 
informative values, it should be kept in mind that their bases on the third and fourth order moments tend to exaggerate the effect of high deviations.

\section{RESULTS AND DISCUSSION}

\subsection{ANOVA: NOEC and LOEC}

Since error is a null-mean variable, in theory any test directly or indirectly based on the Student $t$ distribution can reveal the statistical significance of lower differences than those caused by the standard deviation of the error $(\sigma)$, on condition that a sufficient number of replicates is used. However, as expected, the NOEC and LOEC estimates obtained through ANOVA revealed several problematic aspects, which were reliably quantified by means of simulations using the model (1) with the parametric values specified in Table 1.

The first problem is related to the lack of sensitivity of the method (Figure 1). The least sensitive criterion was to consider the one that was significantly different from the control value according to the Student test- those responses in which the CI lower limit is higher than the CI higher limit of the control value. Despite its reputation of not to be very tolerant with the type I error, the Scheffé test was more discriminative than the Student one. The Dunnett test was the most discriminative, as well as the most tolerant against the decrease in the number of replicates. However, they were all far from detecting the $1 \%$ of the maximum response as significantly different from the control value, even under the most favorable conditions: $\sigma=0.05$ and an infrequent number of ten replicates.

Even if the examination of a numerous and dense dose sequence, each test provided different values of NOEC and LOEC, and -in a logical, but unsatisfactory way- for all of them the distance between both indexes was found statistically not significant. In addition, the curves in Figure 1 showed that even a small increase of the sensitivity would demand a not very reasonable increase of the number of replicates. Thus, the characterization of a toxic agent -as repeated in many legal regulations- through the dose that produces the $10 \%$ of the maximum response seems related to the fact that this value -a not much more refined index than the $\mathrm{EC}_{50}$ in the ecological context-is within the reach of ANOVA.

Independently of the specific form of the DR profile used, it is obvious that those cases with maximum slopes which are closer to the origin produce higher responses to low doses, allowing to obtain a greater precision from the point of view of the dose. However, under the perspective of the response, which is the key variable, the situation is the same with any profile, and a confidence interval cannot be defined for any variable. The most controversial aspect of this method is perhaps the fact that the experimenter can select ad hoc a post hoc test providing an apparent statistical rigor to a wide range of conclusions derived from the same data.

Undoubtedly, the above results represent some of the reasons why many researchers in the ecotoxicological field consider NOEC and LOEC as ambiguous notions which are accepted only unwillingly. Here we want to underline that such an ambiguity tends to underestimate the toxicological risk predictions.

\subsection{DR modeling and some of its properties}

The solution of any function by means of an over-determined mathematical system should satisfy two basic needs: to oppose degrees of freedom to the error and to cover the domain in 
such a way that the regions which are essential to define the parametric values are well represented. In addition, if a minimization of the experimental work is desired, attention should be paid to the distribution of the observations (the design), because none space is homogeneous regarding the information that provides for calculating the parameters of a given function. Thus, for a sigmoidal function as (1), high responses are essential to define the scale parameter $(K)$, intermediate ones to define the localization $(m)$ and shape $(a)$ parameters, and low responses -in a function that lacks of an intercept- provide little information.

The simulations with 2,000 repetitions (Figure 2 and Figure 3) achieved by using the model (1) with different error $(\sigma)$ and number of observations $(N)$ showed that the error affected the parametric estimates in the order $K<m<a$, and tended to produce over-estimation, since the distributions of positive parameters were limited only on the left: $[0, \infty)$. Moreover, when the three estimates were significant $(\alpha=0.05)$ in $100 \%$ of the repetitions, their CI showed to depend on $\sigma$ and $N$ according to very regular trends. For a constant $\sigma$, CI decrease hyperbolically when $N$ increased; for constant $N$, CI increase linearly with $\sigma$ (this increase becomes hyperbolic, but loses regularity, if the proportion of significant estimates descends below $100 \%$ ).

When the typical experimental error of a given system is known, these regularities allow to develop a tabulation to guide the design to control the CI. The main aspects of this control will be discussed next, and the design-guiding tabulation will be described in Appendix section (Table A2). Although we will suppose a response type $R=f(D)$, all the conclusions are transferable to the type $R=f(\ln D)$, provided that geometric relationships between design and functional profile are conserved.

From now on $n$ is defined as the number of different values of the independent variable (doses, the null one included), $D_{m}$ as the maximum dose $\left(D_{m}=1\right.$ unless otherwise stated), and we suppose the same number of replicates $(r)$ in each dose (hence the number of observations is $N=n \times r$ ). To facilitate comparisons, we will use the notation CI for the $\%$ of the confidence intervals of any parametric estimation $(\theta)$. Thus, the conventional expression $\theta \pm \mathrm{CI}$ means that the $\theta$ estimate is statistically significant only if $\mathrm{CI}<100$.

\subsubsection{Regions of the experimental domain}

The geometric progression of the abscissa (dose) is a typical feature of the DR design because: for a given $n$, it provides the best representation of the regions which are essential to define the parametric estimates. Its advantages are diminished as $n$ increases, but even with $n=25$ it is better than the obvious alternative in linear series. This last progression over-represents the high responses at the expense of the intermediate ones, and therefore it reduces the CI of $K$, but increases those of $m$ and $a$ (Table 1A). As these last parameters are more error-sensitive, in a linear design they lose statistical significance at values of $\sigma$ lower than in the geometric option.

\subsubsection{Over-representation of the low responses}

The same reasons that make suitable the geometric series are opposed to the designs overrepresenting the low responses, in general, a frequent practice that has two justifications. One is to find the interval which will be left to ANOVA for NOEC and LOEC estimations, and another is the expectation of possible stimulatory responses at low levels of agents which are inhibitory at high levels (hormesis). Both justifications can be licit, but both should be excluded from DR analysis when the pertinence of a simple sigmoidal model has been accepted and the purpose is to estimate its parameters minimizing their CI. As in the preceding case, the effect of these designs decreases as $n$ increases (Table 1B), but a design with [25×2, $\left.\mathrm{D}_{1}=0.100\right]$ is still preferable to $\left[25 \times 2, \mathrm{D}_{1}=0.025\right]$. In fact, the addition of observations within the response-region lower than $\sim 10 \%$ of the maximum does not contribute to improve the definition of any parameter. 


\subsubsection{Decreasing functions}

When a toxic causes the decrease of any variable or parameter of the system (biomass, specific growth rate, fertilizing capability or others) from a value $V_{0}$ to $V_{\mathrm{i}}$, a common practice is the direct fitting of the $V_{\mathrm{i}} / V_{0}$ ratio to a decreasing function as, for example, $H=K-1$. It is not advisable, since the parametric $C I$ are always lower if the increasing response $1-\left(V_{\mathrm{i}} / V_{0}\right)$ is fitted to a function such as the model (1).

\subsubsection{Replicates}

Replicates reduce the effects of the experimental error, but do not contribute to improve the representation of the key regions for the parametric estimates (Table 1C). Thus, at least up to $n=10-12$, at equal degrees of freedom the increase of the dose number $n$ is more efficient than the increase of the replicate number $r$ (a $9 \times 2$ design, for example, produces lower CI than a $6 \times 3$ one, and a $22 \times 2$ design is also preferable to $12 \times 4$, despite its lower degrees of freedom). With $n>15$ the difference weakens and even tends to be reversed, since once a sufficient representation of the key regions is attained, the reduction of the error effects gains importance.

\subsubsection{Smoothing}

Smoothing by the moving averages method -as recommended by authors such as Jonczyk et al. (1991) to determine $C E_{50}-$ reduces the parametric CI and provides statistical significance to fittings which would not have it if raw data were used. The disadvantage is the bias promoted by the procedure in some parametric estimates. When the usual smoothing -with a window of three values- is applied to a sigmoidal profile without error, only parameter $a$ is biased, with a slight reduction of the slope (bias is accentuated by higher windows). As this effect is opposite to that error, it does not seem problematic. However, it is obvious that low $n$ values enhance bias, as well as the error sensitivity of $K$. Series $\mathrm{C}$ and $\mathrm{D}$ in Table 1 are illustrative in this respect, and show that, with $n>10$, smoothing produces satisfactory results, reducing in $\sim 40 \%$ the parametric CI without undesirable consequences.

\subsubsection{Towards the optimal design}

An extensive set of simulations carried out with systematic combinations of designs and parametric values led to conclude that, for any type of profile generated with equation (1) and any dose number $(n)$, an appropriate design to optimize simultaneously the accuracy of the three parametric estimates should comply with the following conditions:

C1. The dose series should be performed in geometric progression with ratio $g=\left(D_{m} / D_{1}\right)^{1 /(n-2)}$.

C2. The minimum dose should be that one that produces a response of $\sim 10 \%$ of the maximum one.

C3. The response should present $25-30 \%$ observations that produce asymptotic responses.

The flexible ranges in $\mathrm{C} 2$ and $\mathrm{C} 3$ conditions prevent to identify this design as optimum in absolute terms. However, the parametric CI resulting from these conditions are always a good approximation to their minimum values, allowing to define a quasi-optimum (Q) design. The condition C2 may seem paradoxical, since it avoids low responses despite they are an important purpose of the analysis. However, this is a consequence of the functional form describing the phenomenon, and, as in the Galileo's experiment, it means only that it is not very reasonable to insist on measuring times at short spaces. If the absence of observations on the initial interval worries the experimenter, they can be added, provided that the rest of the design is preserved. They would be more useful in other place, but are not detrimental in this one.

Figure 4 shows three $10 \times 2$ designs derived from these rules, for three responses differing only in the parameter $a$. The central case corresponds to a symmetrical Weibull function $(a=3.26)$, and 
the other two to the limit in which this function turns into the Bertalanffy one $(a=1)$, or, on the contrary, when it get closer to a step function in $m(a=20)$. It should be noticed that, if the central case is taken as a reference, an appropriate representation of the parametric key regions, with the same number of observations, requires to extend the dose domain as $a$ decreases, and in the opposite case to reduce it. It should also be underlined that, although the Q design produces the least CI with any profile, it does not imply that the CI in any profile will be equal (Table 2). The widest $\mathrm{CI}$ correspond to the Bertalanffy profile, and within the $[1 \leq a \leq 50]$ interval, whose upper limit is excessive in practice, the CI of $m$ is strongly reduced as $a$ increases, with a negligible effect on $K$ and $a$ parameters.

This way, the preliminary tests required by any DR analysis allow to approximate to the Q design (see Appendix A), increasing the robustness of the model (1) against the experimental error. Finally, it can be pointed out that the simulation conditions used here were not concessive at all. In fact, many experimental evidences suggest that the usual error in the DR context is heteroscedastic, and higher in the intermediate than in the extreme responses. This would produce less distorting deviations than those found assuming homoscedastic hypothesis, irrespective of whether ordinary or weighed least squares are used for calculation.

Accepting these conditions, we will examine now the contents of this type of models to define less problematic notions than NOEC and LOEC.

\subsection{Relationships between the dose corresponding to a given response and its CI}

When a statistically significant model $(1)$ is achieved in its basic parametric form $\left(m_{0.5}\right.$ as localization parameter), the CI of the dose corresponding to any response $p$ can be calculated by fitting the results to a reparametrized form of (1), in which that parameter is -simply changing the value of $q$ - precisely $m_{\mathrm{p}}$. Figure 5A shows a specific case -not specially satisfactory- among the 2,000 ones simulated using the initial parametric values specified in Table 1 , with $\sigma=0.125$ in a $20 \times 1$ design. The estimates were: $K=0.961 \pm 13.6 \% ; m_{0.5}=0.228 \pm 20.7 \% ; a=1.866 \pm 45.9 \%$, and, once such a solution was obtained, the calculation was repeated for 21 different values of $m_{\mathrm{p}}$ (from $m_{0.01}$ to $m_{0.99}$ ). Results are described next.

The CI of $m_{\mathrm{p}}$ was minimum for $m_{0.5}$ and increased towards lower and higher responses, with tendencies that defined two non-symmetrical branches (Figure 5B). Such an asymmetry is depending on the functional form, and it is conserved even in symmetrical functions $(a=3.26)$. The estimates of $K$ and $a$, as well as their respective CI, were constant for any value of $p$ used for reparametrization. The CI of $m_{\mathrm{p}}$ was $86.9 \%$ for $m_{0.02}$ and $103.7 \%$ for $m_{0.01}$, which means that in this case the results allow to establish doses and confidence intervals down to a value between 1 and $2 \%$ of the maximum response.

In addition, the relationship between the $p$ value in the $m_{\mathrm{p}}$ parameter and the parametric CI could be adjusted, for both branches, to a hyperbolic equation as:

$$
C I(p)=b_{0} \frac{1+b_{1} p}{1+b_{2} p}
$$

where $b_{0}$ is the intercept, and $b_{1}, b_{2}$ are fitting coefficients. It is important to note that the statistical significance of $m_{\mathrm{p}}$ is a consequence -not a cause- of its CI value. Therefore, the descriptive accuracy of the equation (4) is not depending on the significance of $m_{\mathrm{p}}$. In other words: when $p$ decreases, possible non-significant estimates of $m_{\mathrm{p}}$ do not prevent a consistent contribution of their $\mathrm{CI}$ to the definition of the equation (4). 
When each reparametrization was applied to the 2,000 repetitions of an assay, instead of to a single case, the result was the same, apart from an expected difference: $K$ and $a$ estimates, as well as their CI, varied slightly in the successive fittings. This fact, however, did not alter the conclusion drawn from the single case, thus showing the robustness of the relationship (4). Indeed (Figure 5c), this relation began to be distorted only when, in fittings to the basic parametric form, the significance of the three parameters was below $100 \%$.

\subsection{Notion of minimum detectable response (MINDER)}

If in the fitting of an experimental series to the model (1) the three parametric estimates are significant when the localization parameter is $m_{0.5}$, the solution of the equation (4) for the lower response branches is immediate using the CI of a $m_{\mathrm{p}}$ parametric family with decreasing values of $p$. If we want to conserve some limit notion, the minimum detectable response ( $p_{0}$ or MINDER) can be defined now without ambiguity as that corresponds to the concentration ( $m_{\mathrm{p} 0}$ Or MINDERC) whose CI is just $100 \%$ of its value. Such a response is obtained making the first member of (4) equal 100 and isolating $p$ :

MINDER $=p_{0}=\frac{b_{0}-100}{100 b_{2}-b_{0} b_{1}}$

the corresponding concentration being the result of replacing $R$ with $p_{0}$ in the reciprocal form (1B) of the DR model:

MINDERC $=m_{p 0}=m_{0.5}\left\{\ln \left[1-\left(p_{0} / K\right)\right] / \ln 0.5\right\}^{1 / a}$

An intercept $\left(b_{0}\right)$ lesser than 100 means that the model enables to estimate reliably doses and $\mathrm{CI}$ corresponding to any response higher than zero, thus making empty the notion of MINDER, as in the cases with $\sigma=0.05$ and 0.100 in Figure 5c. Although with $\sigma=0.150$ this value could be calculated, it would not be licit to do it, since in the 2,000 repetitions of the fitting to the basic form $\left(m_{0.5}\right)$ of the equation $(1)$, the three parameters were significant with a frequency lower than 100\% (very close: $99.7 \%$; that is why the little distortion of the relation (4)). Finally, with a $\sigma=0.125$ in the single assay (Figure 5B), a well-defined MINDER $(1.1 \%$ of the maximum response) is determined by the abscissa of the ordinate $\mathrm{CI}=100$, with a MINDERC of 0.026 dose units, whose $\mathrm{CI}$ is exactly the same value.

These results demonstrated that the DR analysis not only enables to specify CI for its predictions at low doses, but also it has a much higher resolution power than ANOVA. In the example studied, whose conclusions are generalizable, if we suppose a NOEC determined by ANOVA with $\sigma=0.05$, 10 replicates and the most discriminative test restricted to 3 doses, we would find that DR produces estimates with well-defined CI much lower than NOEC, even under an error of 2.5 times higher and with $66 \%$ of the experimental effort. If such an effort was equaled with a $15 \times 2$ design maintaining $\sigma=0.125$, the parametric CI would descend $30 \%$, emptying of content the notion of MINDERC or, equivalently, producing accurate assessments under $\sigma=0.160$.

\subsection{Notion of enveloping profiles}

The parametric CI enable a second alternative to quantify the reliability of a DR model in its predictions at low responses. A parametric CI implies that, given the variability of the dependent 
variable, the studied phenomenon could turn up in any of the forms described by the parametric family covered by such an interval. This fact is illustrated in Figure 6A-C, using the results of the $20 \times 1(\sigma=0.125)$ design discussed above, for each parameter separately (notice that readings lacking in physical meaning should be avoided: in a death/survival response, for example, it is obvious that the CI of a $K=1$ value does not imply the possibility of a mortality higher than the whole population, but the possibility of a total mortality at doses lower than that predicted by the function with $K=1$ ).

Now then, if we consider the $2^{3}=8$ profiles resulting from combining the upper $(+)$ and lower $(-)$ limits of each parameter with the rest of them (Figure 6D):

$$
\begin{array}{lllllllll}
K: & + & + & + & + & - & - & - & - \\
m: & + & + & - & - & + & + & - & - \\
a: & + & - & + & - & + & - & + & -
\end{array}
$$

we obtain a set in which two enveloping profiles (EP) are defined by the maximum and minimum responses for each dose. These EP mean the limits of the predictive system that results when all the error possibilities revealed by the assay are considered. Thus, it can be admitted that the dose corresponding to a given response could be found in any point of the interval defined by the intersection of the EP with a parallel to the dose axis at the level of such a response (Figure 6F).

Although each EP implies in general two functions, if a response is specified, each limit of the corresponding dose is defined by only one of the eight profiles. Thus, the interval depicted in Figure $6 \mathrm{~F}$-in which, under the safety focus, only the lower limit, defined by the upper EP, has practical interest- can be obtained by including the appropriate parameters into the reciprocal function (1B). If a general solution is desired, each entire EP can be described (as in Figure 6E) with a sum of two equations (1):

$$
R=K_{1}\left\{1-\exp \left[\ln 0.5\left(D / m_{1}\right)^{a_{1}}\right]\right\}+K_{2}\left\{1-\exp \left[\ln 0.5\left(D / m_{2}\right)^{a_{2}}\right]\right\}
$$

whose parameters can be easily calculated by non-linear least squares using the Solver complement of Microsoft Excel spreadsheet. In this option, the dose which, in each EP, corresponds to the specified response, should be numerically obtained, and again Solver provides an immediate method. Thus, to determine the lower limit $\left(D_{\text {low }}\right)$ of the dose corresponding to the response $R_{\mathrm{a}}$ (a limit given by the upper EP of such a response), the procedure consists of:

1) To include the following contents in four cells (A, B, C, D) of the spreadsheet:

A: Value of the response under evaluation $\left(R_{\mathrm{a}}\right)$.

B: An initial arbitrary value $\left(D_{\text {low }}\right)$ for the lower limit of the dose corresponding to $R_{\mathrm{a}}$.

C: The equation [7] describing the upper EP, producing the response $R_{\text {up }}$ as a function of the initial dose $D_{\text {low }}$.

D: The absolute difference $\operatorname{diff}=\left|R_{\mathrm{a}}-R_{\mathrm{up}}\right|$.

2) To execute Solver to minimize the diff value, with $D_{\text {low }}$ as changing cell. It is convenient to use an initial $D_{\text {low }}$ value producing a response $R_{\text {up }}$ close to $R_{\mathrm{a}}$, as well as to include the restriction $D_{\text {low }}>0$.

The same procedure is applied next to calculating the upper limit $\left(D_{\text {up }}\right)$ of the dose corresponding to the response $R_{\mathrm{a}}$, using equation (7) describing the lower EP.

This enveloping profiles criterion and the preceding one of the reparametrizations are two different approaches to the same problem (the reliability of the predictions at low doses), and 
therefore their results, although may be not exactly coincident, should be congruent, as indeed it happens (Figure 7). The use of the EP interval as CI of the dose corresponding to a given response can seem too precautionary, but, in fact, the EP intervals are convergent with the CI obtained by reparametrization as the response descends.

Such a convergence is very precise when $b_{0}<100$ in equation (5) and the notion of MINDERC becomes empty (Figure $7, \sigma=0.100$ ). But since EP exist down to $D=0$, they provide defined intervals even when the reparametrization method reaches a non-significant $m_{\mathrm{p}}$ (that is, when the notion of MINDERC is not empty: Figure $7, \sigma=0.125)$. Under these conditions, the EP interval becomes slightly lower than CI, and the acceptation of the first one as a true CI can be arguable as too concessive. In any case, it is much less concessive than NOEC and LOEC, its margins are well delimited, and any discussion can be argued -contrarily to that occurs with NOEC and LOEC- on the basis of unambiguous values and intervals.

\section{CONCLUSIONS}

It is difficult to add something to the copious criticism that numerous authors have devoted to the notions of NOEC and LOEC, not only conceptually unfortunate, but mediated by a not very sensitive method, whose suitability in toxicological analysis is difficult to hold, especially taking into account the existence of the dose-response modeling. The problem is so obvious that it forces to wonder why these notions hold their apparent validity in the legal regulations of many countries, thus hindering their eradication in the toxicological practice. In our opinion, there are reasons of two different types, sometimes interacting with each other.

The only field -the environmental toxicology- in which these concepts subsist (Jager. 2012) provides a certain evidence about the first type of reasons. Since in conflicts involving environmental risks of toxic agents, the priorities are not always clear, it seems serviceable, to sow doubts in context of legal controversy, a good availability of messy notions as a "maximum dose with non-observable response" at a variable distance of a "minimum dose with observable response", and the added possibility of the existence, below the "non-observable adverse effect level", of an interval with favorable hormetic effects on the observable threshold. This last an idea which is dauntlessly defended in some fora (Calabrese and Baldwin. 2003; Calabrese. 2004; Ellman and Sunstein. 2004), and which has led to suggest the need of relaxing the environmental protection policies (Calabrese. 2004; Teeguarden et al. 2000).

Although it is not the right moment for discussing hormesis (a phenomenon first described by Southam and Ehrlich, 1943) as «a stimulatory effect of subinhibitory concentrations of any toxic substance on any organism»), it seems pertinent to point out two details connecting the hormetic school with some of the issues treated here. One of them is its contribution to a radical distrust towards the DR modeling (Calabrese. 2007), and to the idea of possible unexpected health effects at low doses of deleterious agents, a conjecture in which too many things -from immunity mechanisms to detoxifying enzymatic induction- are mixed. Another is the fact that the experimental basis of this last idea can be very doubtful. Thus, in the assessment of complex mixtures, or responses of a dynamic system disregarding the time -as it is frequent in environmental toxicology- biphasic curves often arise, whose suggestion of favorable effects at low doses is only artifactual (Murado and Vázquez. 2007, 2010).

The second type of reasons above alluded has much to do with the preference, in assessments with possible legal projection, by operative routines with a minimum of calculation exigencies. Such a preference is logical, but can lead to excesses: in the same way that a rigorous toxicological evaluation cannot be claimed when ANOVA is used, a too schematic DR analysis 
does not guarantee the statistical significance of its estimates. Not algebraically explicit models, direct use of profile-decreasing data, over-representation of low responses, poorly defined asymptotes, inopportune smoothing and insufficient observations, sometimes giving priority to the replicates over the appropriate coverage of the domain, are common problems in the bibliography of the field, which penalize -strongly, if they are associated- the efficiency of the DR analysis. And so, when, after a perhaps laborious assay, the results do not allow to quantify the statistical reliability of the model, frustration can promote the temptation of going back to the comfortable ANOVA, in which these questions simply do not emerge.

Nevertheless, in many of the abundant ecotoxicological results that are produced per year, any of the two proposed methods can be applied to provide statistical criteria which facilitate the abandonment of NOEC and LOEC. Indeed, if the worst comes to the worst, MINDERC represents the point in which LOEC and NOEC should converge, thus replacing both concepts with a single, unambiguous limit, based on the calculation of confidence intervals which are ignored by those notions, and with the additional advantage of a very superior sensitivity. In the general case, it will be possible to specify, without ambiguity, the CI of the dose corresponding to whatever response we decide to consider.

Finally, the use of the table Q (see Table A2) we propose in Appendix allows to define the DR design producing, with a good approximation, the least parametric CI under the work conditions we decide to establish. Of course, the reparametrizations and enveloping interval methods can be applied under any conditions; table Q (Table A2) only facilitates the selection of a design which avoids unexpected results.

\section{ACKNOWLEDGEMENTS}

Authors wish to thank Ministerio de Ciencia e Innovación (project CTM2010-18411, FEDER funds from European Union) for financial support. Miguel Angel Prieto Lage was awarded one grant from the JAE predoctoral program financed by the CSIC. 


\section{FIGURE CAPTIONS}

\section{Main manuscript}

Figure 1. Percent of responses that are significantly different $(\alpha=0.05)$ from the control, as a function of the dose, according to the Student (left), Scheffé (center) and Dunnett (right) tests, for $\sigma=0.05$ (up) and $0.10\left(\right.$ down), using $3(\bigcirc), 6(\triangle)$ and $10(\nabla)$ replicates. Doses are given as multiples of the value $\left(\mathrm{D}_{0.01}\right)$ producing $1 \%$ of the maximum response. Simulations were carried out by using the model (1) with the parametric values specified in Table 1. Determination of NOEC and LOEC for different number of replicates is illustrated on the central upper case.

Figure 2. Parametric estimates and their $\mathrm{CI}$ in designs with increasing number of doses and two replicates, supposing constant error of $\sigma=0.10$ (up), and under constant $(30 \times 2)$ design with increasing error (down). Simulations as in Figure 1.

Figure 3. Distributions of the parametric estimates in simulations with $\sigma=0.10$ and $10 \times 2$ (up) and $20 \times 2$ (down) designs. Vertical dotted lines mark the true parametric values (specified in Table 1).

Figure 4. Q designs (points) for the specified profiles (dotted lines). Numerical results with $\sigma=0.10$ in Table 2. Note the different dose domains.

Figure 5. A: a single case from a simulation with the parameters specified in Table $1, \sigma=0.125$ and $20 \times 1$ design parameter (points) and its fitting to the equation (4) (lines). C: as B, but supposing values of 0.05 $(\diamond)$, B: variation of the CI of the parameter $m_{\mathrm{p}}$ as a function of the response $p$ specified for such a 0.10 $(\bigcirc)$ and $0.15(\bullet)$ for $\sigma$. For details, see text.

Figure 6. A, B, C: limits of the parametric families defined when considering individually the parameters $K, m, a$ with their respective CI. D: profiles of the 8 possible combinations of lower and upper parametric values as defined by their CI. E: enveloping profiles of the set depicted in D (points), and their fittings to the additive model (7) (lines). F: method of the enveloping interval. For details, see text.

Figure 7. Horizontal series represent values and CI of the doses corresponding to different responses, according to the reparametrizations $(\bigcirc)$ and the enveloping interval $(\bullet)$ methods. Simulations with the model from Table 1 and the $\sigma$ values specified on graph.

\section{Appendix}

Figure A1: CI of the parameter $m$ as simultaneous functions of $a$ and $n$ for $\sigma=0.05$ (left), and of $\sigma$ and $a$ for $n=30$ (right). In both cases points are values from Table Q, and lines the corresponding fittings to the equations (A2\&3) and (A $3 \& 4)$. 


\section{TABLE CAPTIONS}

\section{Main manuscript}

Table 1. Parametric estimates obtained by applying the model (1) to the results of simulations (2,000 repetitions) carried out under some conditions with illustrative value. $\mathrm{Y} / \mathrm{N}$ : smoothed/raw data; G/L: doses in geometric/lineal series; IC: average confidence interval as percentage of the estimate value; SS: percentage of statistically significant estimates (underlined if $\mathrm{SS}<100$ ); SK and KT: skewness and kurtosis coefficients; ALL SS: percentage of fittings in which all the estimates were statistically significant; $r^{2}$ : correlation coefficient between observed and predicted values. True parametric values: $K=1.000, m=0.250, a=2.000$. See text for details.

Table 2. Results obtained by applying Q-designs to the simulations of three sigmoidal profiles differing in the $a$ parameter. In all the cases $K=1.0$ and $m=0.350 . \mathrm{D}_{1}$ and $\mathrm{D}_{\mathrm{m}}$ : minimum and maximum dose values. Rest of notations as in Table 1. See text for details.

\section{Appendix}

Table A1: Called as table QD, show examples of Q-designs for $n, a, m$ values.

Table A2: Called as Table Q, it shows the parametric CI in Q designs for 1, 2, 3 and 4 replicates. Notice that, in each box, $\mathrm{CI}(K)$ and $\mathrm{CI}(a)$ are the same for each $\mathrm{CI}(\mathrm{m})$ column located above them. 


\section{REFERENCES}

Box GEP, Muller ME. Note on the Generation of Random Normal Deviates. Ann Math Statist 1958;29:610-611.

Calabrese EJ. Threshold Dose--Response Model--RIP: 1911 to 2006. Bioessays 2007;29:686-8.

Calabrese EJ, Baldwin LA. Toxicology rethinks its central belief. Nature 2003;421:691-2.

Calabrese EJ. Hormesis: a revolution in toxicology, risk assessment and medicine. EMBO Rep 2004;5:S37-40.

Chapman PM, Caldwell RS, Chapman PF. A warning: NOECs are inappropriate for regulatory use. Environmental Toxicology and Chemistry 1996;15:77-9.

De Lean A, Munson PJ, Rodbard D. Simultaneous analysis of families of sigmoidal curves: application to bioassay, radioligand assay, and physiological dose-response curves. Am J Physiol 1978;235:E97-102.

Ellman LM, Sunstein CR. Hormesis, the precautionary principle, and legal regulation. Hum Exp Toxicol 2004;23:601-11.

Fox DR, Billoir E, Charles S, Delignette-Muller ML, Lopes C. What to do with NOECS/NOELS? prohibition or innovation?. Integrated Environmental Assessment and Management 2012;8:764-6.

Gompertz B. On the Nature of the Function Expressive of the Law of Human Mortality, and on a New Mode of Determining the Value of Life Contingencies . Phil Trans R Soc Lond 1825;115:513-583.

Jager T. Bad habits die hard: The NOEC's persistence reflects poorly on ecotoxicology. Environmental Toxicology and Chemistry 2012;31:228-9.

Kooijman S. An alternative for NOEC exists, but the standard model has to be abandoned first. Oikos 1996;75:310-316.

Kooijman S. Parametric analyses of mortality rates in bioassays. Water Res 1981;15:107-119.

Kooijman SALM, Bedaux JJM. Some statistical properties of estimates of no-effect concentrations. Water Res 1996;30:1724-8.

Lera S, Macchia S, Pellegrini D. Standardizing the Methodology of Sperm Cell Test with Paracentrotus Lividus. Environ Monit Assess 2006;122:101-9.

Murado MA, González MP, Vázquez JA. Dose-response relationships: An overview, a generative model and its application to the verification of descriptive models. Enzyme Microb Technol 2002;31:439-55.

Murado MA, Vázquez JA. The notion of hormesis and the dose-response theory: A unified approach. J Theor Biol 2007;244:489-99. 


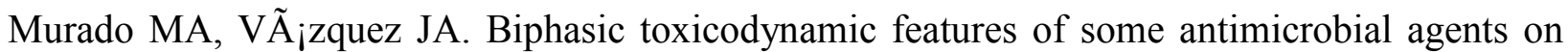
microbial growth: a dynamic mathematical model and its implications on hormesis. BMC microbiology 2010;10:220-34.

Murrell JA, Portier CJ, Morris RW. Characterizing Dose-Response I: Critical Assessment of the Benchmark Dose Concept. Risk Analysis 1998;18:13-26.

Negri AP, Heyward AJ. Inhibition of coral fertilisation and larval metamorphosis by tributyltin and copper. Mar Environ Res 2001;51:17-27.

Novelli AA, Losso C, Ghetti PF, Ghirardini AV. Toxicity of heavy metals using sperm cell and embryo toxicity bioassays with Paracentrotus lividus (Echinodermata: Echinoidea): comparisons with exposure concentrations in the Lagoon of Venice, Italy. Environ Toxicol Chem 2003;22:1295-301.

Pires AM, Branco JA, Picado A, Mendonça E. Models for the estimation of a ?no effect concentration?. Environmetrics 2002;13:15-27.

Prieto MA, Murado MA, Vázquez JA, Anders Y, Curran TP. A new microplate procedure for simultaneous assessment of lipophilic and hydrophilic antioxidants and pro-oxidants, using crocin and $\beta$-carotene bleaching methods in a single combined assay: Tea extracts as a case study. Food Res Int 2013.

Prieto MA, Rodríguez-Amado I, Vázquez JA, Murado MA. $\beta$-Carotene Assay Revisited. Application To Characterize and Quantify Antioxidant and Prooxidant Activities in a Microplate. J Agric Food Chem 2012.

Prikler S. Robert de Levie: Advanced Excel for scientific data analysis, 2nd ed. Analytical and Bioanalytical Chemistry 2009;395:1945.

Riobó P, Paz B, Franco JM, Vázquez JA, Murado MA, Cacho E. Mouse bioassay for palytoxin. Specific symptoms and dose-response against dose-death time relationships. Food and Chemical Toxicology 2008a;46:2639-47.

Riobó P, Paz B, Franco JM, Vázquez JA, Murado MA. Proposal for a simple and sensitive haemolytic assay for palytoxin: Toxicological dynamics, kinetics, ouabain inhibition and thermal stability. Harmful Algae 2008b;7:415-29.

Southam CM, Ehrlich J. Effects of Extract of western red-cedar heartwood on certain wooddecaying fungi in culture. Phytopathology 1943;33:517-524.

Teeguarden JG, Dragan Y, Pitot HC. Hazard assessment of chemical carcinogens: the impact of hormesis. J Appl Toxicol 2000;20:113-20.

Vázquez JA, González MP, Murado MA. Effects of lactic acid bacteria cultures on pathogenic microbiota from fish. Aquaculture 2005;245:149-61.

Warne M, Van Dam R. NOEC and LOEC data should no longer be generated or used. Australasian Journal of Ecotoxicology 2008;14:1-5.

Weibull W. A statistical distribution function of wide applicability. Journal of Applied Mechanics 1951;18:293-7. 
Xie Z, Wong N, Qian P, Qiu J. Responses of polychaete Hydroides elegans life stages to copper stress. Marine Ecology-progress Series 2005;285:89-96. 
$\mathrm{T}$ able 1. Parametric estimates obtained by applying the model (1) to the results of simulations $(2,000$ repetitions) carried out under some conditions with illustrative value. $\mathrm{Y} / \mathrm{N}$ : smoothed/raw data; G/L: doses in geometric/lineal series; IC: average confidence interval as percentage of the estimate value; SS: percentage of statistically significant estimates (underlined if SS<100); SK and KT: skewness and kurtosis coefficients; ALL SS: percentage of fittings in which all the estimates were statistically significant; $r^{2}$ : correlation coefficient between observed and predicted values. True parametric values: $K=1.000, m=0.250, a=2.000$. See text for details.

\begin{tabular}{|c|c|c|c|c|c|c|c|c|c|c|c|c|}
\hline \multirow{4}{*}{$\begin{array}{l}\text { smoothing } \\
\text { D series } \\
D_{1}\end{array}$} & \multicolumn{2}{|c|}{ A } & \multicolumn{2}{|c|}{ B } & \multicolumn{4}{|c|}{$\mathrm{C}$} & \multicolumn{4}{|c|}{$\mathrm{D}$} \\
\hline & $\mathrm{N}$ & $\mathrm{N}$ & $\mathrm{N}$ & $\mathrm{N}$ & $\mathrm{N}$ & $\mathrm{N}$ & $\mathrm{N}$ & $\mathrm{N}$ & $\mathrm{Y}$ & $\mathrm{Y}$ & $\mathrm{Y}$ & $\mathrm{Y}$ \\
\hline & G & $\mathrm{L}$ & G & G & G & G & G & G & G & G & G & G \\
\hline & 0.100 & 0.100 & 0.100 & 0.025 & 0.100 & 0.100 & 0.100 & 0.100 & 0.100 & 0.100 & 0.100 & 0.100 \\
\hline$n \times r$ & $25 \times 2$ & $25 \times 2$ & $25 \times 2$ & $25 \times 2$ & $6 \times 4$ & $8 \times 3$ & $12 \times 2$ & $24 \times 1$ & $6 \times 4$ & $8 \times 3$ & $12 \times 2$ & $24 \times 1$ \\
\hline$\sigma$ & 0.190 & 0.190 & 0.150 & 0.150 & 0.130 & 0.130 & 0.130 & 0.130 & 0.130 & 0.130 & 0.130 & 0.130 \\
\hline$K$ & 1.008 & 1.006 & 1.004 & 1.010 & 1.007 & 1.007 & 1.007 & 1.008 & 0.998 & 0.996 & 1.003 & 1.007 \\
\hline CI $(\%)$ & 12.1 & 9.4 & 9.2 & 11.3 & 11.7 & 12.1 & 12.0 & 12.2 & 10.9 & 9.1 & 7.6 & 6.7 \\
\hline SS (\%) & 100.0 & 100.0 & 100.0 & 100.0 & 100.0 & 100.0 & 100.0 & 100.0 & 100.0 & 100.0 & 100.0 & 100.0 \\
\hline SK & 1.32 & 1.45 & 0.43 & 0.85 & 0.20 & 0.75 & 0.44 & 0.73 & 0.53 & 0.66 & 0.77 & 0.82 \\
\hline KT & 5.92 & 7.57 & 0.33 & 4.44 & 0.31 & 1.60 & 0.82 & 2.04 & 1.03 & 1.31 & 1.68 & 2.95 \\
\hline$m$ & 0.252 & 0.251 & 0.251 & 0.253 & 0.253 & 0.253 & 0.254 & 0.253 & 0.250 & 0.249 & 0.251 & 0.252 \\
\hline CI (\%) & 17.0 & 18.4 & 13.0 & 16.5 & 18.3 & 18.2 & 17.4 & 17.2 & 18.4 & 14.3 & 11.1 & 9.4 \\
\hline SS (\%) & 100.0 & 99.9 & 100.0 & $\underline{99.9}$ & 100.0 & $\underline{99.9}$ & 100.0 & 100.0 & 99.9 & 100.0 & 100.0 & 100.0 \\
\hline SK & 1.39 & $\overline{0.31}$ & 0.33 & $\overline{1.19}$ & 0.17 & $\overline{0.62}$ & 0.62 & 0.77 & $\overline{0.77}$ & 0.77 & 0.82 & 0.84 \\
\hline KT & 7.25 & 0.94 & 0.52 & 7.59 & 0.63 & 1.67 & 0.97 & 1.93 & 1.70 & 1.65 & 2.71 & 2.16 \\
\hline$a$ & 2.074 & 2.120 & 2.046 & 2.049 & 2.079 & 2.076 & 2.089 & 2.062 & 1.529 & 1.828 & 1.971 & 2.052 \\
\hline CI $(\%)$ & 37.8 & 46.8 & 29.5 & 33.1 & 41.8 & 40.4 & 39.3 & 38.1 & 30.3 & 28.4 & 23.9 & 20.9 \\
\hline SS (\%) & 100.0 & 100.0 & 100.0 & 100.0 & 100.0 & 100.0 & 100.0 & 100.0 & 100.0 & 100.0 & 100.0 & 100.0 \\
\hline SK & 1.55 & 2.28 & 0.69 & 0.98 & 0.82 & 0.82 & 1.06 & 0.83 & 0.43 & 0.50 & 0.41 & 1.02 \\
\hline KT & 8.10 & 11.97 & 1.09 & 1.95 & 1.32 & 1.11 & 2.62 & 1.75 & 0.67 & 0.58 & 0.22 & 3.14 \\
\hline ALL SS (\%) & 100.0 & 99.9 & 100.0 & 99.9 & 100.0 & 99.9 & 100.0 & 100.0 & 99.9 & 100.0 & 100.0 & 100.0 \\
\hline$r^{2}$ & 0.782 & 0.749 & 0.852 & $0 . \overline{880}$ & 0.917 & $0 . \overline{909}$ & 0.902 & 0.894 & $0 . \overline{947}$ & 0.950 & 0.961 & 0.965 \\
\hline
\end{tabular}


Table 2. Results obtained by applying Q-designs to the simulations of three sigmoidal profiles differing in the $a$ parameter. In all the cases $K=1.0$ and $m=0.350 . \mathrm{D}_{1}$ and $\mathrm{D}_{\mathrm{m}}$ : minimum and maximum dose values. Rest of notations as in Table 1. See text for details.

\begin{tabular}{|c|c|c|c|c|c|c|}
\hline$a$ & \multicolumn{2}{|c|}{1.0} & \multicolumn{2}{|c|}{3.259} & \multicolumn{2}{|c|}{20.0} \\
\hline $\mathrm{R}$ at $\mathrm{D}_{1}$ & \multicolumn{2}{|c|}{0.100} & \multicolumn{2}{|c|}{0.100} & \multicolumn{2}{|c|}{0.100} \\
\hline & \multicolumn{2}{|c|}{0.0532} & \multicolumn{2}{|c|}{0.1963} & \multicolumn{2}{|c|}{0.3185} \\
\hline & \multicolumn{2}{|c|}{9.875} & \multicolumn{2}{|c|}{0.975} & \multicolumn{2}{|c|}{0.414} \\
\hline$n \times r$ & \multicolumn{2}{|c|}{$10 \times 2$} & \multicolumn{2}{|c|}{$10 \times 2$} & \multicolumn{2}{|c|}{$10 \times 2$} \\
\hline$\sigma$ & \multicolumn{2}{|c|}{0.100} & \multicolumn{2}{|c|}{0.100} & \multicolumn{2}{|c|}{0.100} \\
\hline smoothing & $\mathrm{N}$ & $\mathrm{Y}^{\mathrm{a}}$ & $\mathrm{N}$ & $\mathrm{Y}^{\mathrm{a}}$ & $\mathrm{N}$ & $\mathrm{Y}^{\mathrm{a}}$ \\
\hline K & 1,001 & 0,997 & 1,003 & 0,996 & 1,004 & 0,998 \\
\hline CI $(\%)$ & 8,6 & 5,7 & 8,7 & 5,8 & 8,7 & 5,8 \\
\hline SS (\%) & 100,0 & 100,0 & 100,0 & 100,0 & 100,0 & 100,0 \\
\hline SK & 0,25 & 0,63 & 0,28 & 0,41 & 0,18 & 0,18 \\
\hline KT & 0,37 & 1,90 & 0,23 & 0,87 & $-0,01$ & 0,06 \\
\hline$m$ & 0,355 & 0,351 & 0,352 & 0,350 & 0,350 & 0,350 \\
\hline CI $(\%)$ & 28,9 & 19,7 & 9,0 & 6,2 & 1,5 & 1,0 \\
\hline SS (\%) & 100,0 & 100,0 & 100,0 & 100,0 & 100,0 & 100,0 \\
\hline SK & 0,39 & 0,81 & 0,22 & 0,38 & $-0,09$ & 0,09 \\
\hline KT & 0,10 & 1,33 & 0,30 & 0,40 & 0,03 & 0,09 \\
\hline$a$ & 1,034 & 0,933 & 3,334 & 3,010 & 20,718 & 18,457 \\
\hline CI (\%) & 34,2 & 21,2 & 34,2 & 21,6 & 34,5 & 21,4 \\
\hline SS (\%) & 100,0 & 100,0 & 100,0 & 100,0 & 100,0 & 100,0 \\
\hline SK & 0,72 & 0,30 & 0,50 & 0,45 & 1,16 & 0,27 \\
\hline KT & 1,32 & $-0,08$ & 0,27 & 0,56 & 3,75 & $-0,07$ \\
\hline ALL SS (\%) & 100,0 & 100,0 & 100,0 & 100,0 & 100,0 & 100,0 \\
\hline$r^{2}$ & 0,947 & 0,978 & 0,947 & 0,977 & 0,946 & 0,977 \\
\hline
\end{tabular}




\section{FIGURES}
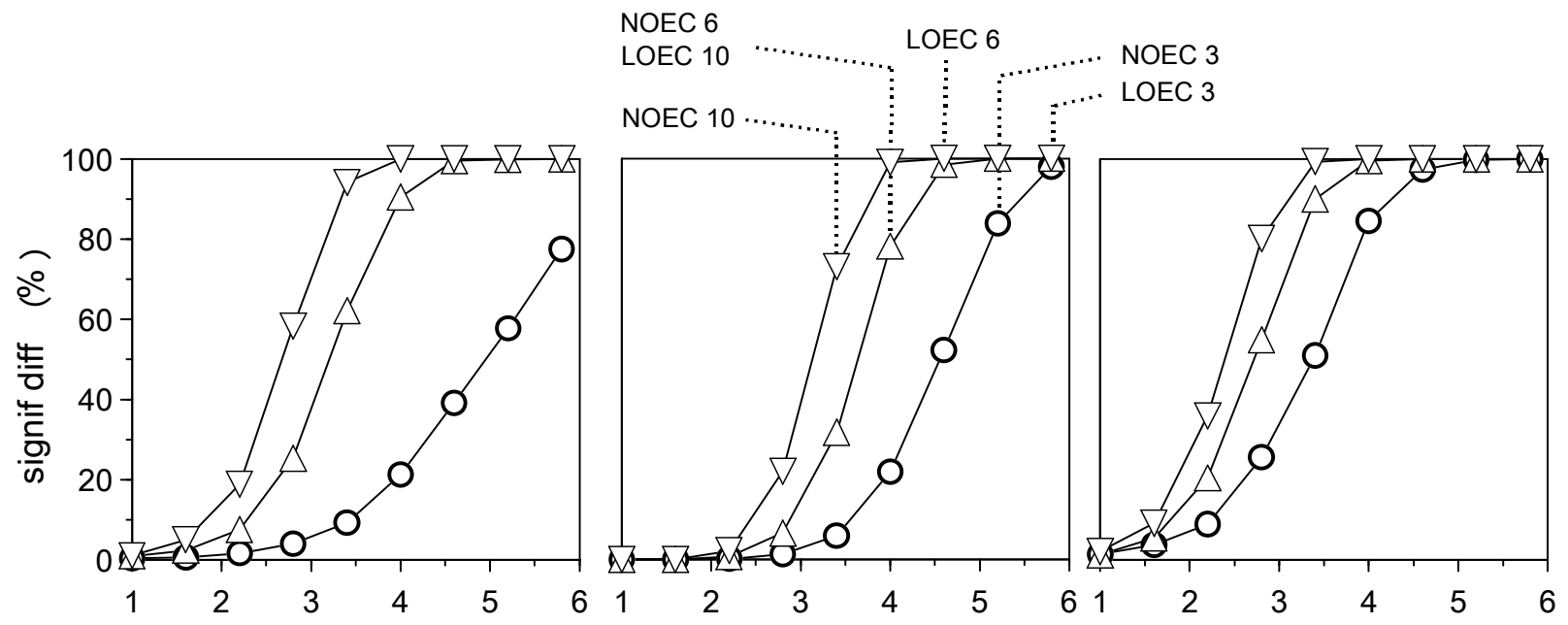

$n \times \mathrm{D}_{0.01}$
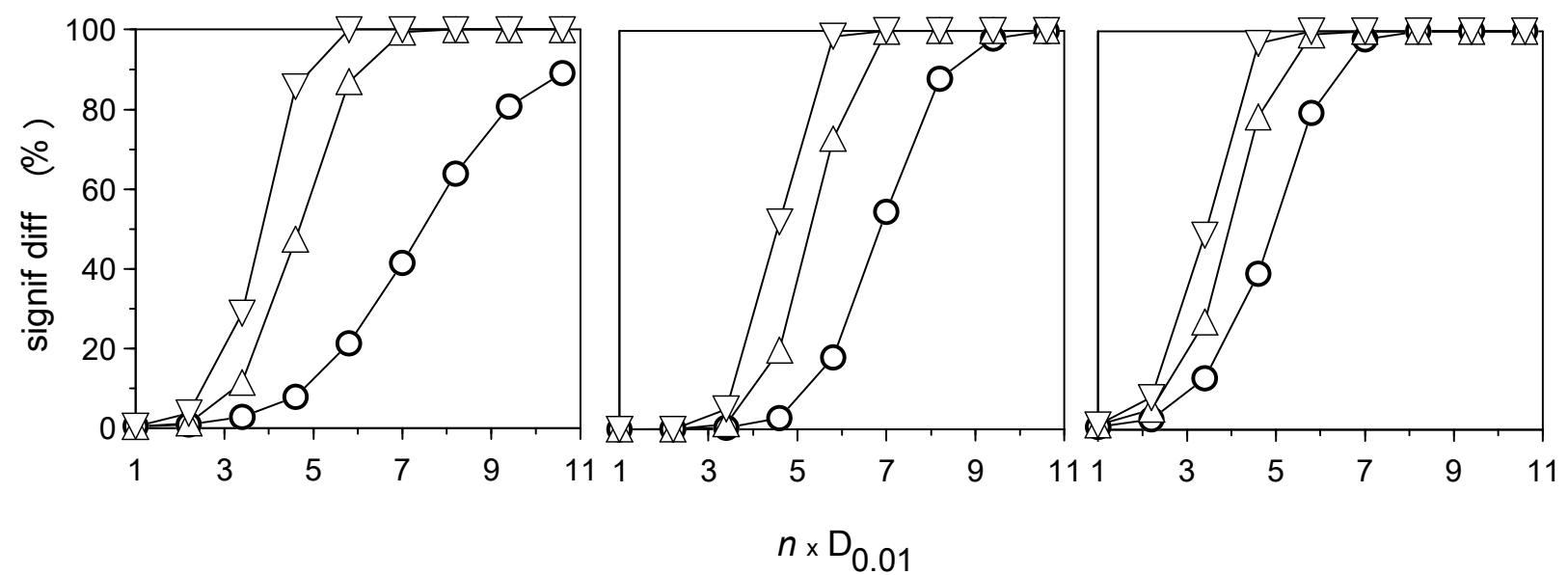

Figure 1. Percent of responses that are significantly different $(\alpha=0.05)$ from the control, as a function of the dose, according to the Student (left), Scheffé (center) and Dunnett (right) tests, for $\sigma=0.05$ (up) and 0.10 (down), using $3(\bigcirc), 6(\triangle)$ and $10(\nabla)$ replicates. Doses are given as multiples of the value $\left(\mathrm{D}_{0.01}\right)$ producing $1 \%$ of the maximum response. Simulations were carried out by using the model (1) with the parametric values specified in table 1. Determination of NOEC and LOEC for different number of replicates is illustrated on the central upper case. 

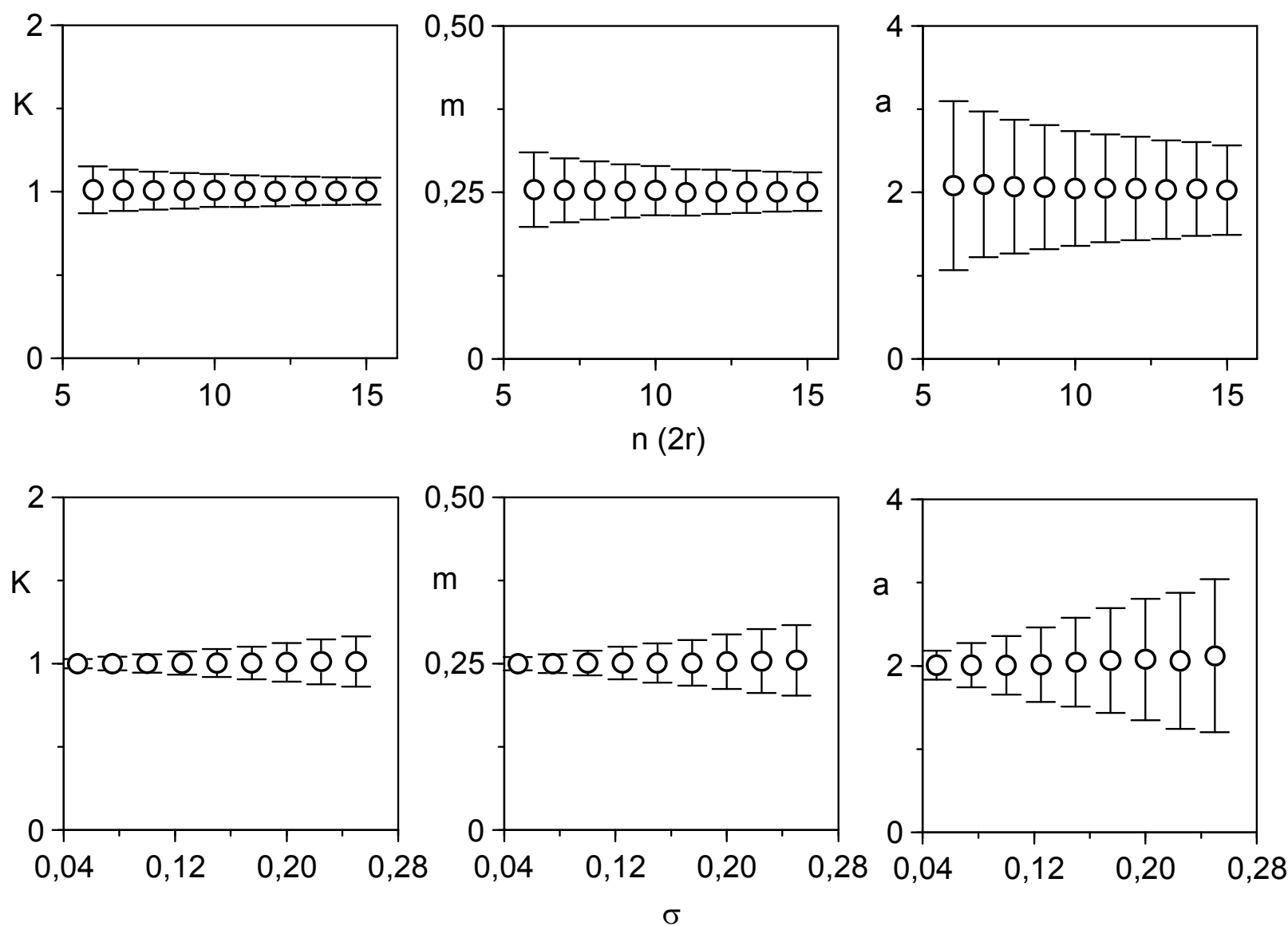

Figure 2. Parametric estimates and their $\mathrm{CI}$ in designs with increasing number of doses and two replicates, supposing constant error of $\sigma=0.10$ (up), and under constant $(30 \times 2)$ design with increasing error (down). Simulations as in figure 1. 

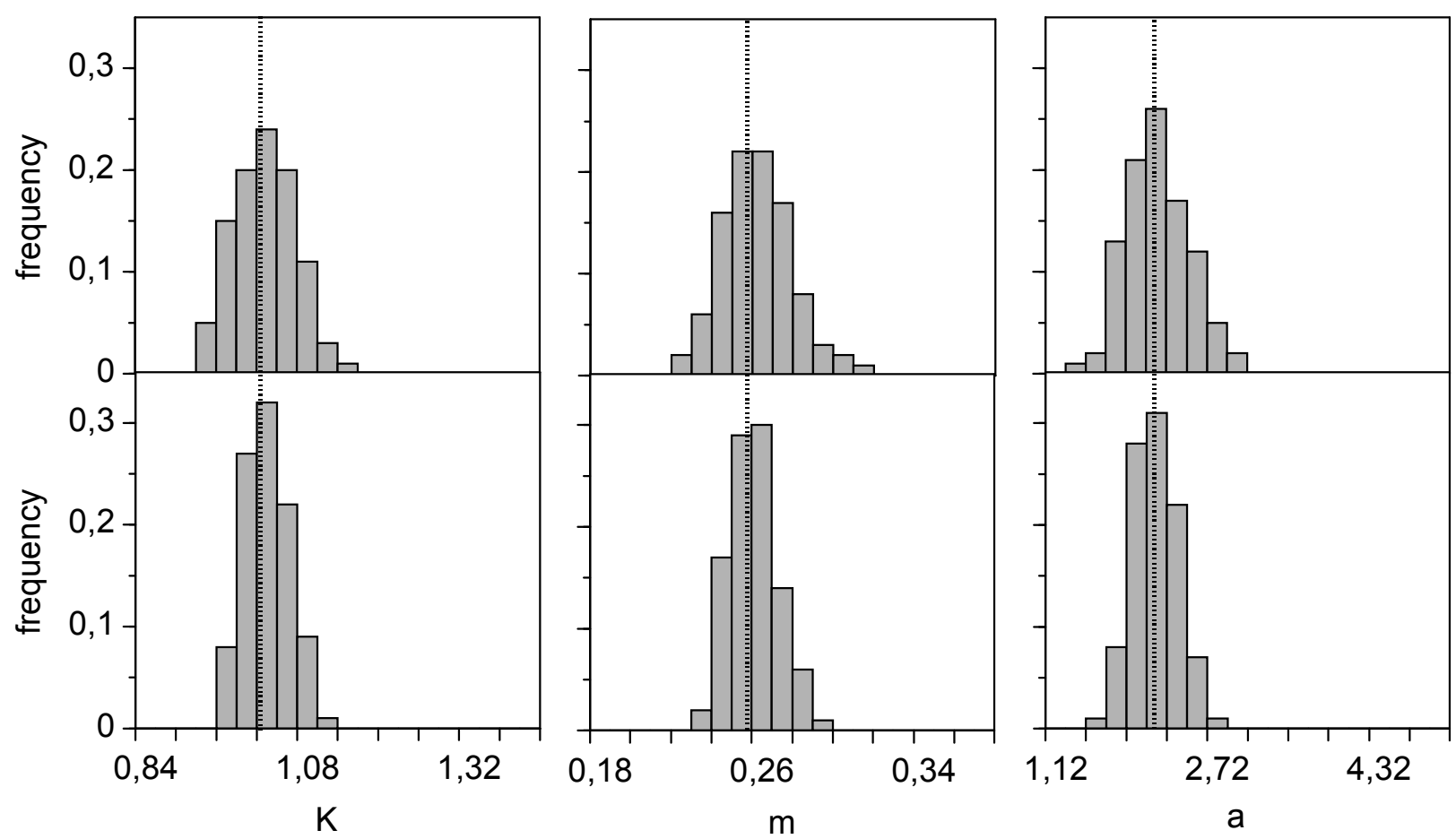

Figure 3. Distributions of the parametric estimates in simulations with $\sigma=0.10$ and $10 \times 2$ (up) and $20 \times 2$ (down) designs. Vertical dotted lines mark the true parametric values (specified in table 1). 


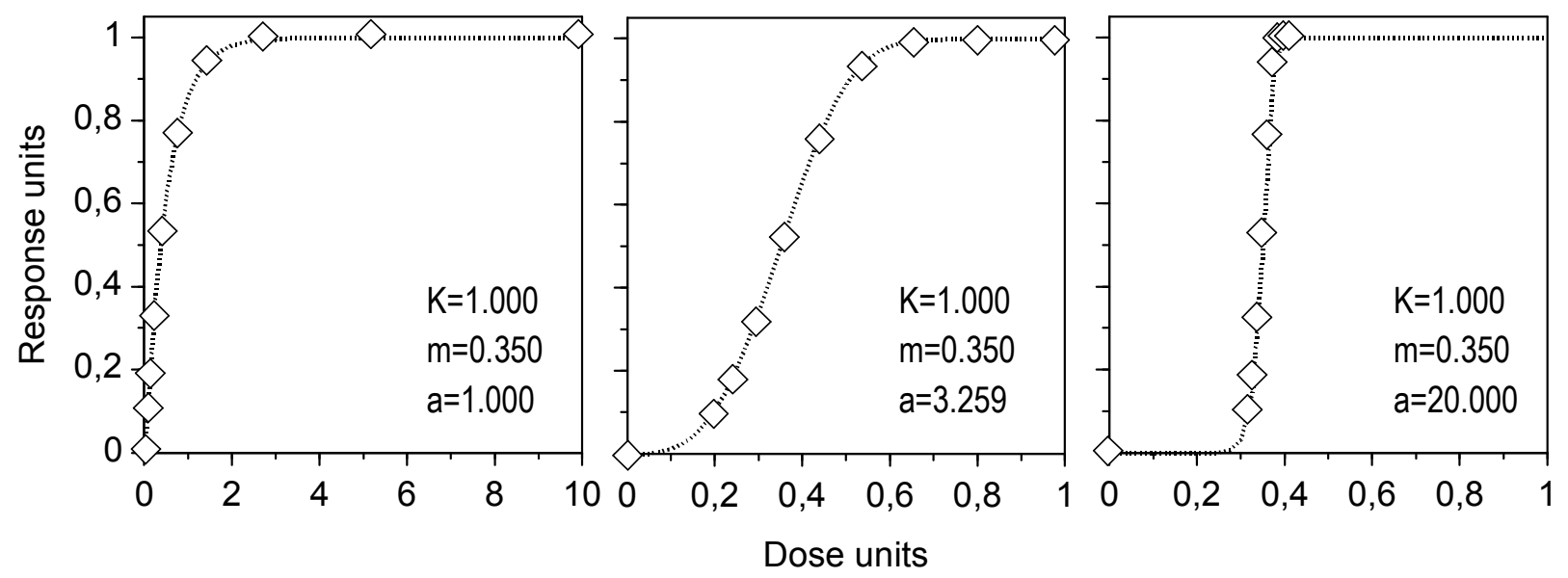

Figure 4. Q designs (points) for the specified profiles (dotted lines). Numerical results with $\sigma=0.10$ in table 2 . Note the different dose domains. 

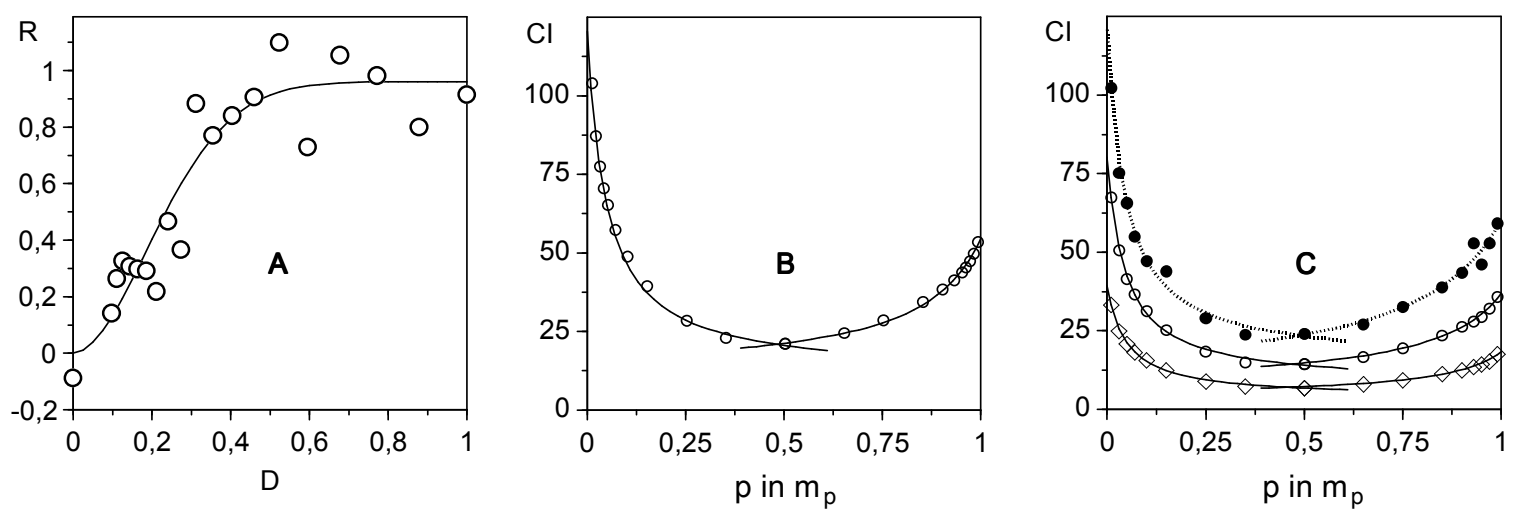

Figure 5. A: a single case from a simulation with the parameters specified in table $1, \sigma=0.125$ and $20 \times 1$ design parameter (points) and its fitting to the equation (4) (lines). C: as B, but supposing values of $0.05(\diamond)$, B: variation of the CI of the parameter $m_{\mathrm{p}}$ as a function of the response $p$ specified for such a $0.10(\bigcirc)$ and $0.15(\bullet)$ for $\sigma$. For details, see text. 

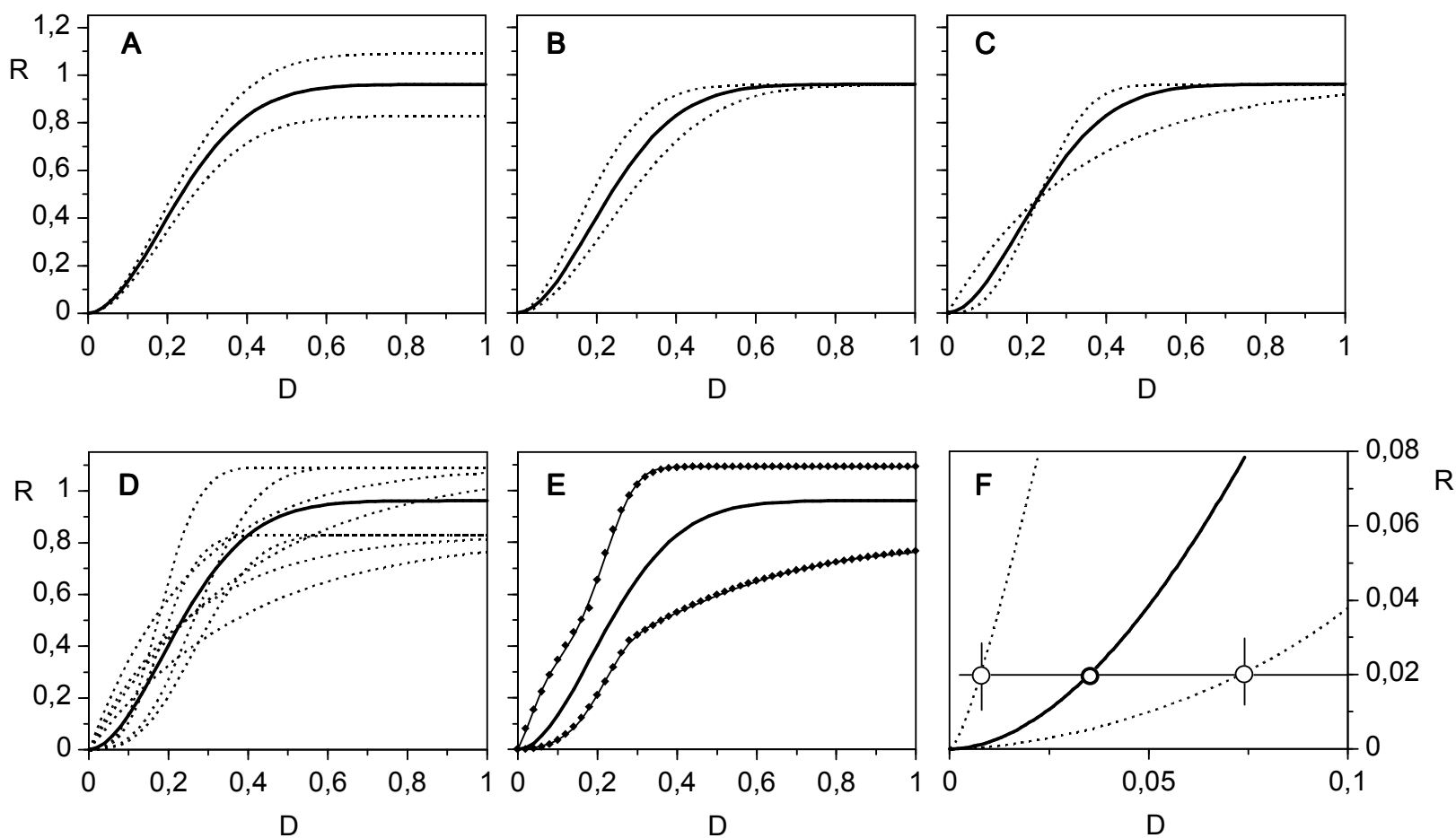

Figure 6. A, B, C: limits of the parametric families defined when considering individually the parameters $K, m, a$ with their respective CI. D: profiles of the 8 possible combinations of lower and upper parametric values as defined by their CI. E: enveloping profiles of the set depicted in D (points), and their fittings to the additive model (7) (lines). F: method of the enveloping interval. For details, see text. 

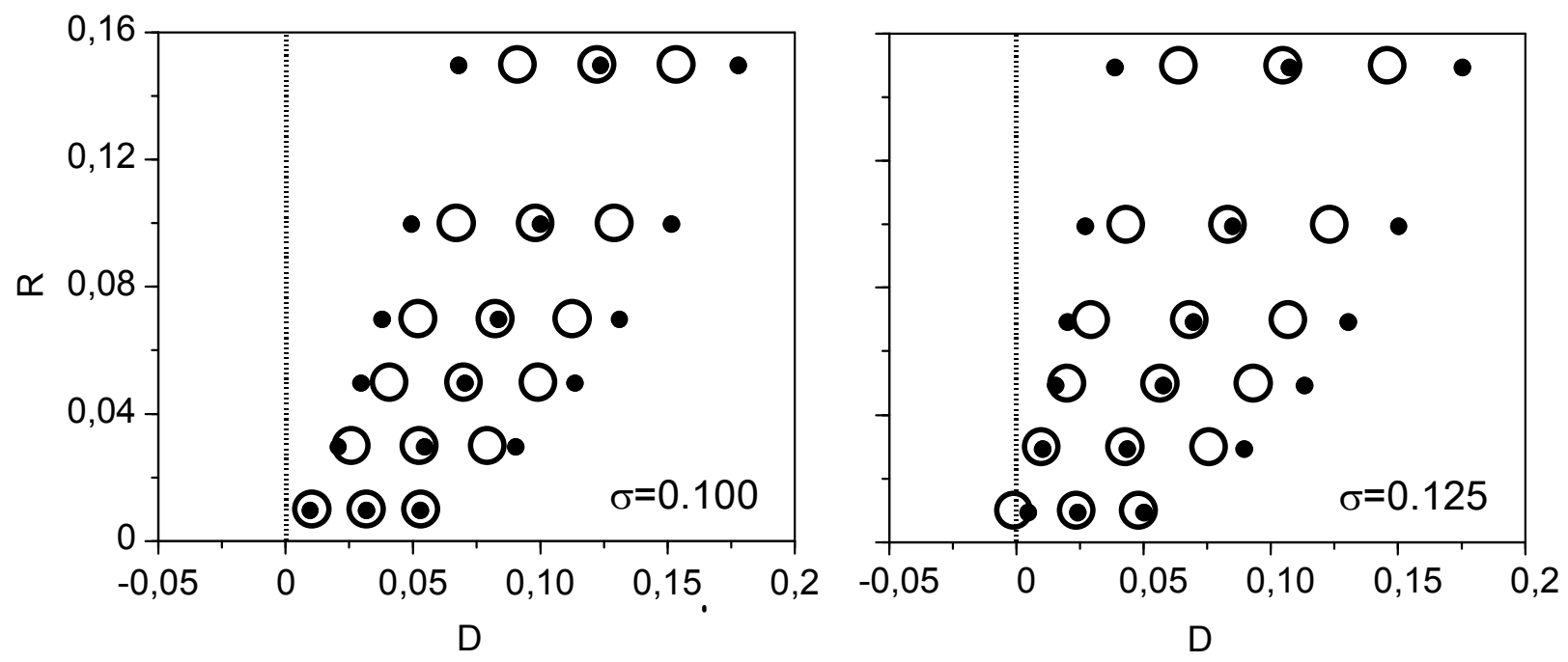

Figure 7: Horizontal series represent values and CI of the doses corresponding to different responses, according to the reparametrizations $(O)$ and the enveloping interval $(\bullet)$ methods. Simulations with the model from table 1 and the $\sigma$ values specified on graph. 


\section{APPENDIX}

\section{A design for achieving a good approximation to the least parametric CI}

The CI of the parametric estimates depend on the error, the functional profile (parametric values) and the experimental design. Since error and profile are a posteriori data, a tabulation of the regularities of this system seems a tedious and fruitless task. However, the DR assays are usually preceded by preliminary tests which provide information about the experimental error, as well as a first approximation to the parametric values of the model that will describe the response. This information, besides the properties of the Q design (as defined by the conditions $\mathrm{C} 1$ to $\mathrm{C} 3$ specified in results 2.5 ) can be easily used to formulate an assay substantially more accurate than that derived from intuitive considerations.

For a given error $(\sigma)$ and number of observations $(N=n \times r)$, a Q design has the following properties:

q1. It leads with a good approximation to parametric estimates with least CI.

q2. $\quad \mathrm{CI}$ are conserved if $K$ and $\sigma$ vary in equal proportion. For example, other conditions being equal, the CI obtained with $K=1, \sigma=0.10$ and with $K=0.5, \sigma=0.05$ are the same.

q3. A Q design for given $\sigma$ and $n$ depends on the parametric values $a$ and $m$. But for a same $a$ value, the $\mathrm{Q}$ designs corresponding to any value of $m$ produce equal $\mathrm{CI}$. The reciprocal statement is not true: for a same $m$ value, the Q designs corresponding to different values of $a$ do not produce equal CI.

\section{Routine formulation of a $Q$ design for a given profile}

In view of the property $\mathrm{q} 2$, the parameter $K$ can be disregarded, and the design defined as a function of the parameters $a$ and $m$, as well as the number of doses $n$ (null included and replicates excluded). Under these conditions, we need to determine the first non-null dose $\left(D_{1}\right)$ and the ratio $(g)$ of the dose progression. It requires the following definitions:

$D_{1} \quad$ First non-null dose. We accept it corresponds to the response $R=0.100$, and it is determined with the equation (1B):

$$
D_{1}=m(\ln 0.9 / \ln 0.5)^{1 / a}
$$

$D_{\mathrm{A}} \quad$ First dose whose response can be considered asymptotic. We accept it corresponds to $R=0.995$, and it is equally determined with the equation (1B):

$$
D_{A}=m(\ln 0.005 / \ln 0.5)^{1 / a}
$$

$A \quad$ Number of doses with asymptotic response: from $D_{\mathrm{A}}$ to the maximum dose $\left(D_{\mathrm{m}}\right)$, both included. It is formulated as the default integer of $30 \%$ of $n$ :

$$
A=\operatorname{INT}(0.3 \times n) ; \text { e.g.: if } n=15, A=4
$$

$Z \quad$ Number of doses into the interval covering from the null one to $D_{\mathrm{A}}$, both included:

$$
Z=n-A+1
$$

$g \quad$ Ratio of the dose progression. It is defined through the $D_{\mathrm{A}} / D_{1}$ quotient, and it is used next to determine the series from $D_{1}$ to the maximum dose $D_{\mathrm{m}}$ :

$$
g=\left(D_{A} / D_{1}\right)^{1 /(Z-2)}
$$

Thus, the entire dose series of a Q design for given values of $n, a$ and $m$ is:

$0, D_{1} \times g^{0}, D_{1} \times g^{1}, D_{1} \times g^{2}, \ldots D_{\mathrm{A}}=D_{1} \times g^{\mathrm{Z}-2}, \ldots D_{\mathrm{m}}=D_{1} \times g^{\mathrm{n}-2}$ 
These definitions enable the immediate preparation, in a spreadsheet, of the formulation that provides the $\mathrm{Q}$ design for whatever conditions we decide (allowing also to try variations in the definitions of $D_{1}$ and $A$ ). The table QD (see Table A1) is only a succinct example to illustrate the use of the decision derived from the examination of a preliminary test in the light of table $\mathrm{Q}$.

Thus, let us suppose that such a test, with coded doses into the $[0,1]$ interval, showed an error with $\sigma \sim 0.15$ and suggested approximate values of $m \sim 0.4$ and $a \sim 3$ for the model (1). If we decide the definitive assay with $n=16$, the dose progression will be $D_{1}=0.213 ; g=1.126$. And in such a case, the expected CI will be the corresponding ones to $\sigma \sim 0.15, n=16, a=3$ in table Q, for the selected number of replicates. It should be noticed that to maintain the initial correspondence between natural and coded values, the first ones will abide now to $\left[0, D_{\mathrm{m}}=1.122\right]$ coded interval.

\section{The table Q}

The steps followed for constructing the table Q (see Table A2) were: 1) definition of the Q designs for an arbitrary value of $m$, combining $a=1,2,3,4,6,8,10,15$ with $n=8,10,12,14,16$, $18,22,30 ; 2$ ) use of each of 64 designs in a virtual assay with 1,000 repetitions (enough for stable results), combining $\sigma=0.050-(0.025)-0.200$ with $r=1,2,3,4$.

CI values included into the table were produced by simulations in which all the parametric estimates were significant in $99.5 \%$ of the 1,000 repetitions. The criterion seems very strict, but it guarantees not only the success of the assays achieved according to the described rules, but also a high regularity, which enables precise interpolations. The equations in this regard that we propose next are merely empirical. They provide excellent fittings (Figure A1), but do not have a special meaning. We will denote in general as $\theta$ one or more of the three parameters $(K, m, a)$ and as $c_{\theta \mathrm{i}(\mathrm{F})}$ the coefficients of the equations describing the relationships between the $\mathrm{CI}$ of $\theta$ and one of the factors ( $F=a, n$ or $\sigma$ ) determining its value. The basic relationships are the following:

1. For constant $\sigma$ :

1.1. The CI of $K$ and $a$ are constant for all $a$, and decrease hyperbolically as $n$ increases:

$C I_{\theta}(n)=c_{\theta 0(n)} \frac{1+c_{\theta 1(n)} n}{1+c_{\theta 2(n)} n} ;(\theta=K, a)$

1.2. The CI of $m$ decrease hyperbolically as $n$ or $a$ increase:

$$
\begin{aligned}
& C I_{m}(n)=c_{m 0(n)} \frac{1+c_{m 1(n)} n}{1+c_{m 2(n)} n} \\
& C I_{m}(a)=c_{m 0(a)} \frac{1+c_{m 1(a)} a}{1+c_{m 2(a)} a}
\end{aligned}
$$

2. For increasing $\sigma$ and constant $n$ and $a$, all parametric CI increase linearly:

$$
C I_{\theta}(\sigma)=c_{\theta 0(\sigma)}+c_{\theta 1(\sigma)} \sigma ;(\theta=K, m, a)
$$

The equations (A1) to (A4) solve any interpolation need when the appropriate series of the table $\mathrm{Q}$ (Table A2) is used as basis of the fitting. 
To illustrate the structure of the table, let us consider now, in the option $r=1$, the CI of $m$ (the more complex case) under two perspectives: as a simultaneous function of $n$ and $a$ for $\sigma=0.05$ (combining (A2) and (A3), and of $\sigma$ and $a$ for $n=30$ (combining (A3) and (A4)):

$$
\begin{aligned}
& C I_{m}(n, a)=c_{m 0(n, a)} \frac{1+c_{m 1(n, a)} n}{1+c_{m 2(n, a)} n} \times \frac{1+c_{m 1(n, a)} a}{1+c_{m 2(n, a)} a} \\
& C I_{m}(\sigma, a)=\left(c_{m 0(\sigma, a)}+c_{m 1(\sigma, a)} \sigma\right) \times \frac{1+c_{m 1(\sigma, a)} a}{1+c_{m 2(\sigma, a)} a}
\end{aligned}
$$

The fittings of the values from table Q (Table A2) to these equations (Figure A1) allow to appreciate the above mentioned regularity, and to justify the omission of the data corresponding to the highest value of $\sigma$ for the three lowest values of $a$ into the table. Such a omission means that in these cases the three parameters are simultaneously significant with a frequency lower than $99.5 \%$, what produces CI values deviated from predicted by the equation (A3\&4).

In fact, the gaps of the tables Q part 1 and 2 correspond to conditions which are not considered appropriate for a successful assay because, in the 2,000 repetitions, at least one of the following results was obtained:

i) At least one parameter with a $\mathrm{CI} \geq 100$, at least in $0.5 \%$ of the cases. Omission is maintained even if all the CI averages were lesser than 100 .

ii) At least one parameter with $\mathrm{CI}$ average higher than 100 , at least in $0.1 \%$ of the cases.

The second result is typical at high values of the parameter $a$, close to the tolerance limits to the $\sigma$ increase and $n$ decrease. In such cases the effect is due to outliers with very low frequency (lesser than $0.5 \%$ ), which are accepted by the macro automatism, but that would be debugged in an intelligent analysis. However, we have preferred to adopt a strict criterion, leaving to the election of the experimenter its possible relaxation, by using the equations (A1) to (A4) for moderate extrapolations. 


\section{APPENDIX TABLES}

Table A1: Called as table QD, show examples of Qdesigns for $n, a, m$ values.

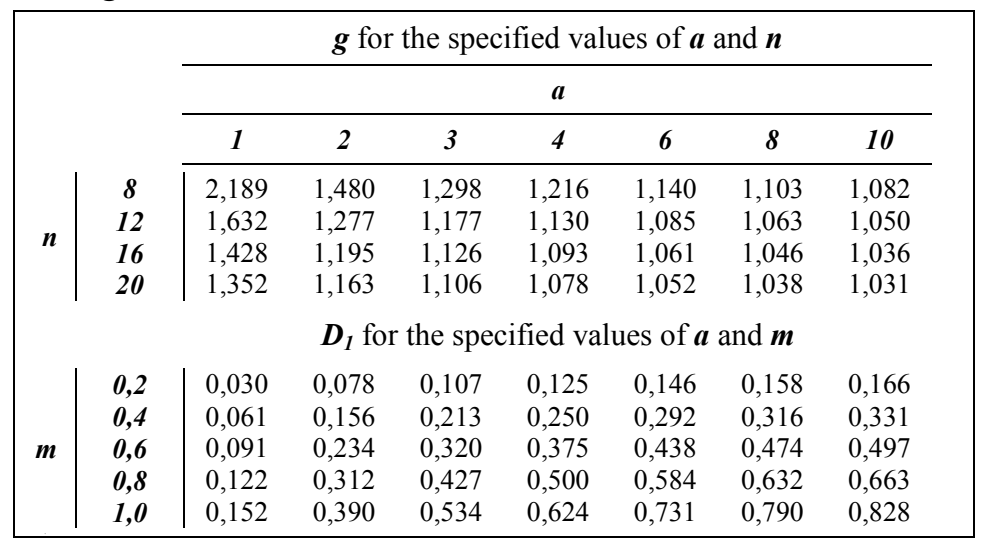


Table A2: Called as Table Q, it shows the parametric CI in Q designs for 1, 2, 3 and 4 replicates. Notice that, in each box, $\mathrm{CI}(K)$ and $\mathrm{CI}(a)$ are the same for each $\mathrm{CI}(m)$ column located above them.

\begin{tabular}{|c|c|c|c|c|c|c|c|c|c|c|c|c|c|c|c|c|c|c|c|c|c|c|c|c|c|c|}
\hline & & & & & $r=$ & & & & & & & & $r=$ & & & & & & & & $r=$ & & & & & \\
\hline$\sigma$ & $a^{n}$ & 8 & 10 & 12 & 14 & 16 & 18 & 22 & 30 & 8 & 10 & 12 & 14 & 16 & 18 & 22 & 30 & 8 & 10 & 12 & 14 & 16 & 18 & 22 & 30 & 8 \\
\hline 0,050 & 1 & 27,9 & 22,4 & 19,4 & 17,4 & 15,9 & 14,8 & 13,1 & 10,9 & 19,5 & 16,1 & 13,8 & 12,2 & 11,3 & 10,5 & 9,2 & 7,7 & 15.9 & 13.0 & 11.1 & 10.1 & 9.1 & 8.6 & 7.6 & 6.3 & \\
\hline & 2 & 13,5 & 11,2 & 9,7 & 8,7 & 7,9 & 7,5 & 6,5 & 5,5 & 9,6 & 8,1 & 6,8 & 6,2 & 5,7 & $|5,3|$ & 4,6 & $\mid \begin{array}{l}\mid \\
\mid\end{array}$ & 7.9| & 6.5 & 5.6 & 5.0 & 4.6 & 4.3 & 3.8 & 3.2 & 6,9 \\
\hline & 3 & 9,2 & 7,5 & $6 \mid$ & 5,8 & | $5,4 \mid$ & $5,0 \mid$ & $4,4 \mid$ & 3,6 & 6,6 & 5,2 & $4,6 \mid$ & 4,1 & 3,7 & 3,5 & 3,1 & $2,6 \mid$ & 5.3 & $|4.3|$ & 3.8 & 3.4 & 3.1 & 2.8 & \begin{tabular}{|l|}
$\mid 2.5$ \\
$\mid$
\end{tabular} & 2.1 & 4,5 \\
\hline & 4 & 6,9 & 5,8 & 4,9 & 4,4 & 4,0 & 3,7 & $|3,3|$ & 2,7 & $|4,8|$ & 4,0 & $|3,5|$ & $|3,1|$ & 2,9 & $\mid 2,6$ & 2,3 & $|1,9|$ & 4.0 & $\mid$\begin{tabular}{|l|}
$\mid$ \\
$\mid$
\end{tabular} & 2.8 & 2.5 & 2.3 & 2.1 & 1.9 & 1.6 & 3,5 \\
\hline & 6 & 4,6 & 3,8 & 3,2 & 3,0 & $\mid 2,7$ & 2,5 & 2,2 & 1,8 & 3,3 & 2,6 & 2,3 [ & 2,1 & 1,9 & 1,7 & 1,6 & 1,3 & 2.6 & 2.1 & 1.9 & 1.7 & 1.5 & 1.4 & 1.3 & 1.1 & 2,3 \\
\hline & 8 & 3,4 & 2,8 & 2,5 & 2,2 & 2,0 & 1,9 & 1,6 & 1,4 & 2,4 & 2,0 & $|1,7|$ & 1,6 & 1,4 & $\mid 1,3$ & 1,2 & 1,0 & $2.0 \mathrm{I}$ & 1.6 & $\mid 1.4$ & 1.3 & 1.2 & 1.1 & 0.9 & 0.8 & 1,7 \\
\hline & 10 & 2,8 & 2,2 & 2,0 & 1,8 & 1,6 & 1,5 & 1,3 & 1,1 & 1,9 & 1,6 & 1,4 & 1,2 & 1,1 & 1,1 & 0,9 & 0,8 & 1.6 & 1.3 & 1.1 & 1.0 & 0.9 & $0.9^{\circ}$ & 0.8 & 0.6 & 1,4 \\
\hline & 15 & 1,9 & 1,5 & 1,3 & 1,2 & 1,1 & 1,0 & 0,9 & 0,7 & 1,3 & 1,1 & 0,9 & 0,8 & 0,8 & 0,7 & 0,6 & 0,5 & 1.0 & 0.9 & 0.8 & 0.7 & 0.6 & 0.6 & 0.5 & 0.4 & 0,9 \\
\hline & $\mathrm{Cl}(\mathrm{K})$ & 9,0 & 6,7 & $\begin{array}{l}6,3 \\
\end{array}$ & \begin{tabular}{|l|}
5,3 \\
\end{tabular} & 5,1 & 4,6 & 4,0 & 3,2 & 6,3 [ & 4,7 & 4,4 & $|3,7|$ & 3,6 & 3,2 & 2,9 & 2,3 & 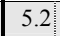 & 3.8 & 3.6 & 3.1 & 3.0 & 2.6 & $|2.3|$ & 1.9 & 4,5 [ \\
\hline & Cl (a) & 31,2 & 26,3 & 22,6 & 20,6 & 18,6 & 17,6 & 15,5 & 13,2 & 22,0 & 18,7 & 15,9 & 14,6 & 13,2 & $12,4] 1$ & 11,0 & 9,4 & 17.9 & 15.1 & 13.0 & 12.0 & 10.8 & 10.2 & 9.0 & 7.6 & 15,6 \\
\hline 0,075 & 1 & & 33,4 & 29,3 & 26,1 & 24,1 & 22,1 & 19,7 & 16,4 & 30,0 & 23,7 & 20,8 & 18,8 & 17,2 & 15,8 & 14,0 & 11,6 & 23.9 & 19.5 & 17.0 & 15.3 & 13.8 & 12.7 & 11.4 & 9.5 & 20,4 \\
\hline & 2 & 21,3 & 16,7 & 15,0 & 13,3 & 12,1 & 11,2 & 9,8 & 8,3 & 14,9 & 11,9 & 10,4 & 9,3 & 8,5 & 7,8 & 6,9 & 5,8 & 11.8 & 9.7 & 8.4 & 7.6 & 7.0 & 6.4 & 5.6 & 4.7 & 10,4 \\
\hline & 3 & 14,0 & 11,4 & 9,9 & 8,7 & $|8,1|$ & $7,5 \mid$ & 6,6 & 5,4 & $9,7 \mid$ & 8,0 & 7,0 & 6,2 & 5,6 & 5,2 & 4,6 & $\mid 3,9$ & 7.9| & 6.6 & 5.6 & 5.0 & 4.7 & 4.3 & 3.8 & 3.2 & 6,9 \\
\hline & 4 & 10,6 & 8,3 & 7,5 & 6,6 & 6,0 & 5,5 & 4,9 & 4,1 & 7,4 & 6,1 & 5,2 & 4,6 & 4,3 & 4,0 & 3,5 & 2,9 & 6.0 & 4.9 & 4.2 & 3.8 & 3.4 & 3.2 & 2.8 & 2.4 & 5,3 \\
\hline & 6 & 7,1 & 5,7 & 4,9 & 4,4 & $|4,0|$ & 3,7 & 3,3 & 2,8 & 4,8 & 4,0 & 3,5 & $|3,1|$ & 2,8 & 2,6 & 2,3 & $\mid$\begin{tabular}{|l|}
$\mid$ \\
$\mid$
\end{tabular} & 4.0 & $|3.3|$ & 2.8 & 2.5 & 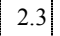 & 2.1 & 1.9 & $\mid 1.6$ & 3,5 \\
\hline & 8 & 5,3 & 4,2 & 3,7 & 3,3 & $\mid 3,0$ & 2,8 & 2,5 & 2,0 & 3,7 & 3,0 & 2,6 & $|2,3|$ & 2,1 & $|2,0|$ & 1,7 & 1,5 & 3.0 & $|2.4|$ & 2.1 & 1.9 & 1.7 & 1.6 & $|1.4|$ & 1.2 & 2,6 \\
\hline & 10 & 4,3 & 3,4 & 2,9 & 2,7 & 2,4 & 2,2 & 2,0 & 1,6 & 2,9 & 2,4 & 2,1 & 1,9 & 1,7 & 1,6 & 1,4 & 1,2 & 2.3 & 2.0 & 1.7 & 1.5 & 1.4 & 1.3 & 1.1 & 1.0 & 2,1 \\
\hline & 15 & 2,9 & 2,3 & 2,0 & 1,8 & 1,6 & 1,5 & 1,3 & 1,1 & 2,0 & 1,6 & 1,4 & 1,2 & 1,1 & 1,1 & 0,9 & : 0,8 & 1.6 & 1.3 & 1.2 & 1.0 & 0.9 & 0.9 & 0.8 & 0.6 & 1,4 \\
\hline & $\mathrm{Cl}(\mathrm{K})$ & 13,9 & $|10,0|$ & \begin{tabular}{|l|}
9,6 \\
\end{tabular} & 8,0 & \begin{tabular}{|l|}
7,7 \\
\end{tabular} & 6,8 & 6,1 & 4,8 & 9,6 & 7,1 & 6,7 & \begin{tabular}{|l|}
5,6 \\
\end{tabular} & $|5,4|$ & $|4,8|$ & 4,3 & \begin{tabular}{|l|l|}
3,4 \\
\end{tabular} & \begin{tabular}{|c|}
7.7 \\
\end{tabular} & \begin{tabular}{|l|l|}
5.8 \\
\end{tabular} & \begin{tabular}{|l|}
5.4 \\
\end{tabular} & 4.6 & \begin{tabular}{|l|}
4.4 \\
\end{tabular} & \begin{tabular}{|l|}
$\mid 3.9$ \\
\end{tabular} & 3.5 & \begin{tabular}{|l|}
2.8 \\
\end{tabular} & \begin{tabular}{|c|}
6,8 \\
\end{tabular} \\
\hline & $\mathrm{Cl}$ (a) & 47,5 & 39,7 & 34,1 & 31,1 & 28,0 & 26,3 & 23,4 & 19,8 & 33,4 & 28,1 & 24,0 & 21,9 & 19,8 & 18,7 & 16,5 & 14,0 & 27.0 & 22.9 & 19.5 & 17.9 & 16.1 & 15.1 & 13.5 & 11.5 & 23,5 | \\
\hline 0,100 & 1 & & & & 35,2 & $\mid 32,1$ & $|29,9|$ & $\mid 26,3$ & 21,9 & & 32,1 & $|27,6|$ & 24,5 & 22,5 & 20,8 & 18,8 & 15,6 & 32.0 & 26.2 & 22.7 & 20.2 & $\mid 18.5$ & $\mid 17.1$ & $|15.1|$ & 12.7 & $|27,7|$ \\
\hline & 2 & & & 19,6 & 17,7 & 16,3 & 14,8 & 13,1 & 11,0 & 20,0 & 16,0 & 13,8 & 12,5 & 11,3 & 10,5 & 9,3 & 7,7 & 16.4 & 13.1 & 11.3 & 10.1 & 9.2 & 8.5 & 7.6 & 6.3 & 14,0 \\
\hline & 3 & & & 13,4 & 11,7 & 11,0 & 9,8 & 8,7 & 7,3 & 13,4 & 10,8 & 9,2 & 8,3 & 7,5 & 6,9 & 6,2 & 5,2 & 10.7 & 8.7 & 7.5 & 6.6 & 6.1 & 5.7 & 5.0 & 4.2 & 9,1 \\
\hline & 4 & & & 9,8 & 9,0 & $\mid 8,0$ & 7,4 & $\mid 6,6$ & 5,5 & $|10,1|$ & 7,9 & 6,9 & 6,2 & 5,7 & 5,3 & 4,6 & 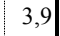 & 8.0 & 6.5 & 5.6 & 5.0 & 4.6 & 4.2 & 3.7 & 3.2 & 7,0 \\
\hline & 6 & & & 6,7 & 5,9 & 5,3 & 5,0 & 4,4 & 3,7 & 6,6 & 5,4 & 4,7 & 4,1 & 3,8 & 3,5 & 3,1 & 2,6 & 5.4 & 4.3 & 3.8 & 3.4 & 3.1 & 2.8 & 2.5 & 2.1 & 4,7 \\
\hline & 8 & & & 5,0 & 4,5 & $|4,1|$ & 3,8 & 3,3 & 2,7 & 4,9 & 4,0 & $|3,5|$ & $|3,1|$ & 2,8 & $|2,6|$ & $|2,3|$ & $\mid$\begin{tabular}{|l|}
1,9 \\
$\mid$
\end{tabular} & 4.0 & \begin{tabular}{|c|}
$\mid$ \\
$\mid$
\end{tabular} .2. & 2.8 & 2.5 & 2.3 & 2.1 & \begin{tabular}{|l|}
$\mid$ \\
$\mid$
\end{tabular} & $\mid 1.6$ & 3,4 \\
\hline & 10 & & & 4,1 & 3,5 & |3,2 & 3,0 & 2,6 & 2,2 & 4,0 & 3,2 & 2,8 & $|2,5|$ & 2,3 & 2,1 & 1,9 & 1,6 & 3.2 & 2.5 & 2.2 & 2.1 & 1.9 & $|1.7|$ & $|1.5|$ & 1.3 & 2,8 \\
\hline & 15 & & & 2,7 & 2,4 & 2,2 & 2,0 & 1,8 & 1,5 & 2,6 & 2,1 & 1,9 & 1,6 & 1,5 & 1,4 & 1,2 & 1,0 & 2.2 & 1.8 & \begin{tabular}{|l|}
1.5 \\
\end{tabular} & 1.4 & 1.2 & 1.1 & 1.0 & 0.9 & 1,8 \\
\hline & $\mathrm{Cl}(\mathrm{K})$ & & & 12,9 & 10,8 & 10,4 & 9,2 & 8,1 & 6,5 & 13,1 & 9,5 & 9,0 & 7,5 & 7,2 & 6,4 & 5,7 & 4,6 & 10.5 & 7.7 & 7.3 & 6.1 & 5.9 & 5.2 & 4.6 & 3.7 & 9,0 \\
\hline & $\mathrm{Cl}(\mathrm{a})$ & & & 45,8 & \begin{tabular}{|l|}
41,9 \\
\end{tabular} & $37,6 \mid$ & 35,3 & $31,2 \mid$ & 26,4 & 45,0 & 37,5 & 32,1 & 29,2 & 26,3 & 24,8 & 22,1 & 18,8 & 36.5 & 30.5 & 26.0 & 23.9 & 21.6 & 20.2 & $\mid 17.8$ & 15.3 & 31,2 \\
\hline 0,125 & 1 & & & & & & $\mid 38,6$ & \begin{tabular}{|l|}
32,8 \\
\end{tabular} & \begin{tabular}{|l|}
27,7 \\
\end{tabular} & & 39,7 & 35,0 & 30,9 & 28,5 & 26,0 & 23,3 & \begin{tabular}{|l|}
19,3 \\
\end{tabular} & & 32.3 & 28.1 & 25.5 & 23.3 & \begin{tabular}{|l|}
21.3 \\
\end{tabular} & \begin{tabular}{|l|}
18.9 \\
\end{tabular} & \begin{tabular}{|l|}
15.9 \\
\end{tabular} & 34,8 \\
\hline & 2 & & & & & 21,1 & 19,8 & 16,3 & 13,8 & & {$[20,3$} & 17,4 & 15,8 & 14,2 & 13,1 & 11,8 & 9,6 & & 16.5 & 14.4 & 12.7 & 11.5 & {$[10.7$} & 9.4 & 8.0 & $\mid 17,3$ \\
\hline & 3 & & & & & 13,6 & 12,6 & 11,0 & 9,2 & & 13,3 & 11,5 & 10,5 & 9,5 & 8,9 & 7,7 & 6,4 & & 10.7 & 9.6 & 8.4 & 7.7 & 7.1 & 6.3 & 5.3 & 11,9 \\
\hline & 4 & & & & & 10,2 & 9,5 & 8,2 & 6,9 & & 10,1 & 8,8 & 7,8 & 7,2 & 6,6 & 5,8 & 4,9 & 10.1 & 8.1 & 7.2 & 6.2 & 5.7 & 5.4 & 4.7 & 4.0 & 8,8 \\
\hline & 6 & & & & 7,5 & 6,9 & 6,2 & 5,6 & 4,6 & & 6,6 & 5,9 & 5,1 & 4,8 & 4,4 & 3,9 & 3,3 & $|6.7|$ & 5.5 & 4.8 & 4.2 & 3.9 & 3.6 & 3.2 & 2.7 & 5,9 \\
\hline & 8 & & & & 5,7 . & 5,3 & 4,8 & 4,1 & 3,4 & & 5,0 & 4,3 & 3,8 & 3,5 & 3,3 & 2,9 & 2,4 & 5.1 & 4.1 & 3.6 & 3.2 & 2.9 & 2.7 & 2.4 & 2.0 & 4,3 \\
\hline & 10 & & & & 4,5 & $|4,1|$ & 3,7 & 3,3 & 2,8 & & 4,0 & $|3,5|$ & $|3,1|$ & 2,9 & $|2,7|$ & 2,3 & $|2,0|$ & & 3.3 & 2.8 & 2.5 & 2.3 & $|2.1|$ & $|1.9|$ & $\mid 1.6$ & 3,5 \\
\hline & 15 & & & & 3,0 & 2,9 & 2,5 & 2,2 & 1,8 & & 2,7 & 2,4 & 2,0 & 1,9 & 1,7 & 1,5 & 1,3 & & 2.2 & 1.9 & 1.7 & 1.5 & 1.4 & 1.3 & 1.1 & 2,3 \\
\hline & $\mathrm{Cl}(\mathrm{K})$ & & & & \begin{tabular}{|l|l|}
13,8 & \\
\end{tabular} & 13,5 & 11,7 & 10,2 & 8,2 & & 11,9 & 11,4 & 9,4 & 9,2 & 8,1 & 7,2 & 5,7 & $\mid 13.3$ & 9.7 & 9.2 & \begin{tabular}{|l|}
7.7 \\
\end{tabular} & \begin{tabular}{ll|}
7.4 \\
\end{tabular} & $\begin{array}{l}6.5 \\
\end{array}$ & 5.8 & $\begin{array}{l}4.7 \\
\end{array}$ & 11,4 \\
\hline & $\mathrm{Cl}$ (a) & & & & 53,4 & 47,8 & 44,7 & 39,2 & 33,3 & & 47,0 & 40,3 & 36,8 & $\mid 33,3$ & 31,1 & 27,6 & 23,4 & 46.0 & 38.4 & 32.8 & 29.8 & 27.0 & 25.3 & 22.4 & 19.1 & $39,7 \mid$ \\
\hline 0,150 & 1 & & & & & & & \begin{tabular}{|l|}
41,1 \\
\end{tabular} & \begin{tabular}{|l|}
33,1 \\
\end{tabular} & & & & $\mid 37,0$ & $\mid 34,5$ & $|31,6| 2$ & \begin{tabular}{|l|}
27,9 \\
\end{tabular} & 23,2 & & & 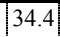 & 30.1 & \begin{tabular}{|l|}
28.0 \\
\end{tabular} & $\mid 26.1$ & \begin{tabular}{|l|}
22.8 \\
\end{tabular} & \begin{tabular}{|l|}
18.9 \\
\end{tabular} & \\
\hline & 2 & & & & & & 23,1 & 20,4 & 16,5 & & & 21,9 & 18,8 & 17,2 & 15,9 & 14,0 & 11,7 & & & 17.0 & 15.1 & 13.9 & 13.0 & ]1.4 & 9.5 & $|21,1|$ \\
\hline & 3 & & & & & & 15,7 & $|13,7|$ & 10,9 & & & $\mid 14,7$ & $\mid 12,5$ & $\mid 11,6$ & 10,7 & 9,3 & 7,7 & & 13.2 & 11.5 & 10.2 & 9.4 & 8.6 & 7.7 & 6.3 & 14,0 \\
\hline & 4 & & & & & & 11,6 & 11,1 & 8,4 & & & 10,9 & 9,3 & 8,6 & 8,0 & 7,0 & 5,8 & & 9.9 & 8.7 & 7.5 & 6.9 & 6.4 & 5.7 & 4.8 & 10,6 \\
\hline & 6 & & & & & & 7,6 & 6,9 & 5,6 & & & 7,1 & 6,4 & 5,9 & 5,3 & 4,6 & 3,9 & & 6.6 & 5.8 & 5.1 & 4.6 & 4.3 & 3.8 & 3.2 & 7,0 \\
\hline & 8 & & & & & & 5,8 & 5,1 & 4,2 & & & 5,4 & 4,7 & 4,4 & 4,0 & 3,5 & 2,9 & & 5.0 & 4.3 & 3.8 & 3.5 & 3.2 & 2.8 & 2.4 & 5,3 [ \\
\hline & 16 & & & & & & & 4,2 & 3,3 & & & 4,3 & 3,8 & 3,5 & 3,2 & 2,8 & 2,3 & & 3.9 & 3.4 & 3.1 & 2.8 & 2.6 & 2.3 & 1.9 & 4,2 \\
\hline & 15 & & & & & & & 2,7 & 2,2 & & & 2,9 & $|2,5|$ & $|2,3|$ & $|2,1|$ & $|1,9|$ & 1,6 & & & \begin{tabular}{|l|}
$\mid$ \\
$\mid$
\end{tabular} & 2.1 & 1.9 & $|1.7|$ & $|1.5|$ & 1.3 & 2,8 \\
\hline & $\mathrm{Cl}(\mathrm{K})$ & & & & & & 14,3 & 12,7 & 9,9 & & & 14,1 & 11,5 & 11,2 & 9,8 & 8,6 & 6,9 & & 11.8 & 11.1 & 9.2 & 9.0 & 7.9 & 7.0 & 5.6 & 13,8 \\
\hline & $\mathrm{Cl}$ (a) & & & & & & 54,5 & 47,8 & 40,1 & & & 49,2 & 44,3 & 40,2 & 37,7 & 33,2 & 28,1 & & 46.3 & 39.5 & 35.9 & 32.4 & 30.6 & 27.1 & \begin{tabular}{|l|}
22.9 \\
\end{tabular} & 47,4 \\
\hline 0,175 & 1 & & & & & & & & 39,0 & & & & & 40,7 & 37,6 & 32,6 & 27,7 & & & & 35.9 & 33.3 & 30.1 & 26.4 & 22.0 & \\
\hline & 2 & & & & & & & & 19 & & & & $|22,2|$ & $|20,6|$ & $|18,7| 1$ & $|16,4|$ & 13,6 & & & & $|17.9|$ & $|16.3|$ & $|15.2|$ & $|13.3|$ & $\mid 11.1$ & \\
\hline & 3 & & & & & & & & 13,3 & & & & 14,9 & 13,6 & 12,3 & 11,0 & 9,0 & & & & 11.9 & 10.9 & 10.2 & 8.9 & 7.4 & \\
\hline & 4 & & & & & & & & 9,7 & & & & 11,2 & 10,4 & 9,3 & 8,2 & | & & & 10.0 & 9.0 & $\mid 8.3$ & 7.5 & 6.7 & 5.5 & \\
\hline & 6 & & & & & & & & 6,6 & & & & 7,7 & 6,8 & 6,2 & 5,5 & 4,5 & & & 6.9 & 5.9 & 5.6 & 5.0 & 4.4 & 3.7 & \\
\hline & 8 & & & & & & & & 4,9 & & & & 5,9 & 5,1 & 4,8 & 4,1 & 3,4 & & & 5.1 & 4.4 & 4.1 & 3.8 & 3.4 & 2.8 & \\
\hline & 10 & & & & & & & 4,8 & 4,0 & & & & 4,5 & 4,0 & $|3,8|$ & 3,3 & 2,7 & & & 4.0 & 3.5 & 3.3 & 3.1 & $2.7 \mid$ & 2.2 & \\
\hline & 15 & & & & & & & & 2,6 & & & & 3,0 & 2,7 & 2,5 & 2,2 & 1,8 & & & 2.8 & 2.4 & 2.2 & 2.0 & 1.8 & 1.5 & \\
\hline & $\mathrm{Cl}(\mathrm{K})$ & & & & & & & 14,9 & \begin{tabular}{|l|l|}
11,7 \\
\end{tabular} & & & & $\begin{array}{l}13,8 \\
527\end{array}$ & 13,2 & 11,5 & 10,2 & $\begin{array}{r}8,0 \\
32\end{array}$ & & & 13.2 & 10.9 & 10.6 & $\begin{array}{r}9.3 \\
958\end{array}$ & $\begin{array}{r}8.2 \\
316\end{array}$ & $\begin{array}{r}6.5 \\
26.7\end{array}$ & \\
\hline & $\mathrm{Cl}$ (a) & & & & & & & 56,4 & 47,2 & & & & 52,7 & 47,1 & 44,23 & 39,3 & 32,9 & & & 46.1 & 42.3 & 38.2 & 35.8 & 31.6 & 26.7 & \\
\hline ,200 & 1 & & & & & & & & & & & & & & & & & & & & & & & & 25.3 & \\
\hline & 2 & & & & & & & & & & & & & & $21,3] 1$ & 18,9 & 15,7 & & & & & & 17.4 & 15.3 & 12 & \\
\hline & 3 & & & & & & & & & & & & & & $|14,4| 1$ & $|12,7|$ & $|10,3|$ & & & & 13.8 & $|12.5|$ & $|11.5|$ & $|10.2|$ & \begin{tabular}{|l|}
$\mid$ \\
$\mid$
\end{tabular} & \\
\hline & 4 & & & & & & & & $\mid 11,7$ & & & & & 11,8 & 10,8 & $|9,4|$ & 7,8 & & & & & $|9.5|$ & $|8.7|$ & $|7.7|$ & \begin{tabular}{|l|}
$\mid$ \\
$\mid$
\end{tabular} & \\
\hline & 6 & & & & & & & & 7,4 & & & & & 8,2 & 7,2 & 6,3 & 5,3 & & & & & 6.3 & 5.9 & 5.1 & 4.2 & \\
\hline & 8 & & & & & & & & 5,7 & & & & & 6,0 & 5,4 & 4,8 & $|3,9|$ & & & & & 4.7 & 4.3 & 3.8 & 3.2 & \\
\hline & 16 & & & & & & & & 4,6 & & & & & & 4,4 & 3,8 & 3,1 & & & & & 3.8 & $3.5^{\circ}$ & 3.0 & 2.5 & \\
\hline & 15 & & & & & & & & 3,0 & & & & & & 2,9 & 2,5 & 2,1 & & & & & 2.5 & 2.3 & 2.1 & 1.7 & \\
\hline & $\mathrm{Cl}(\mathrm{K})$ & & & & & & & & 13,6 & & & & & 15,4 & $|13,4| 1$ & 11,7 & \begin{tabular}{|l|} 
\\
\end{tabular} & & & & 12.6 & & $\mid 10.7$ & $\mid 9.5$ & 7.5 & \\
\hline & & & & & & & & & 54,5 & & & & & 54,5 & 51,44 & 44,8 & 37,8 & & & & 49.1 & 43.6 & 41.2 & 36.2 & 30.6 & \\
\hline
\end{tabular}



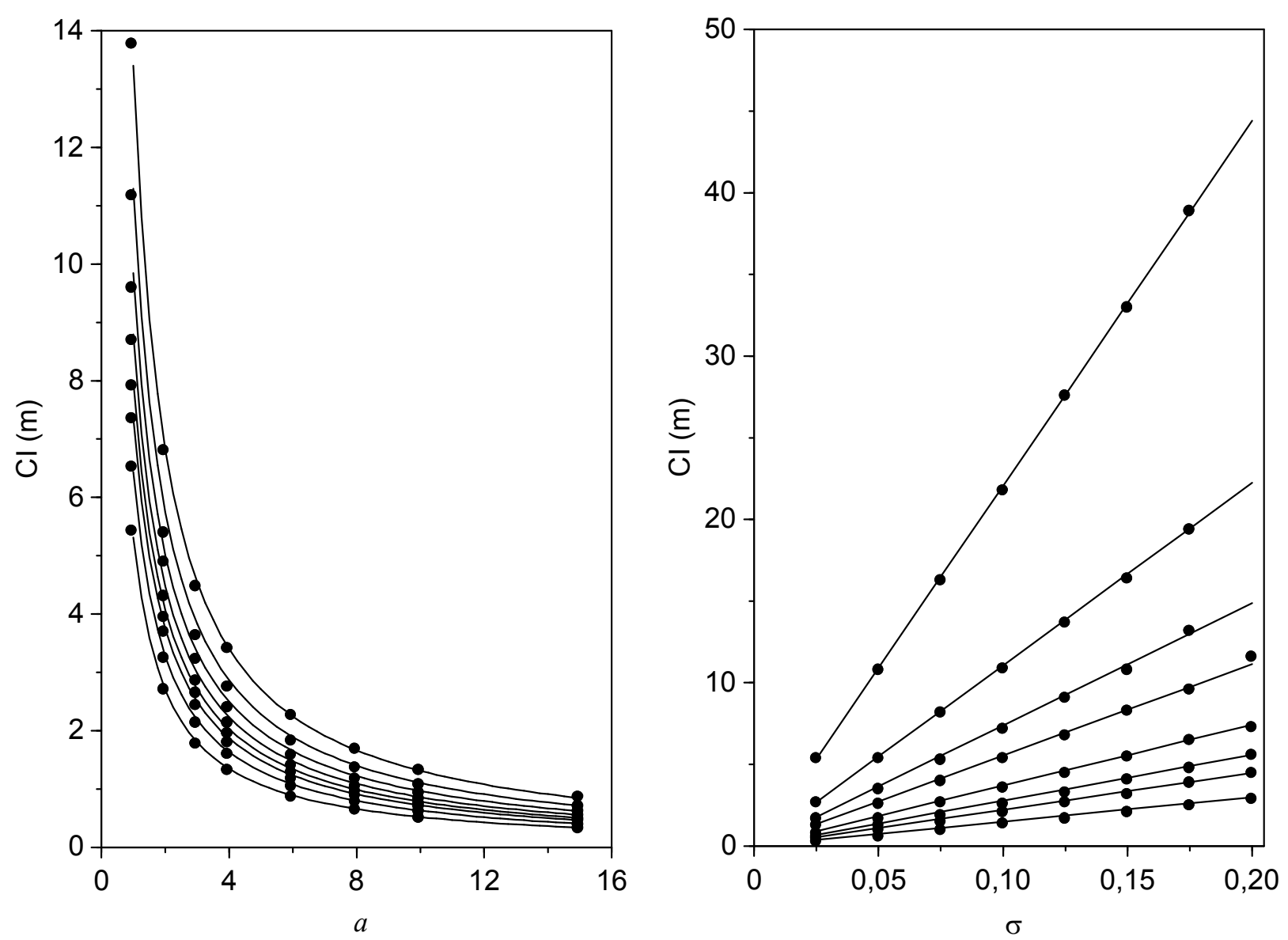

Figure A1: CI of the parameter $m$ as simultaneous functions of $a$ and $n$ for $\sigma=0.05$ (left), and of $\sigma$ and $a$ for $n=30$ (right). In both cases points are values from table Q, and lines the corresponding fittings to the equations $[\mathrm{Q} 2 \& 3]$ and $[\mathrm{Q} 3 \& 4])$. 